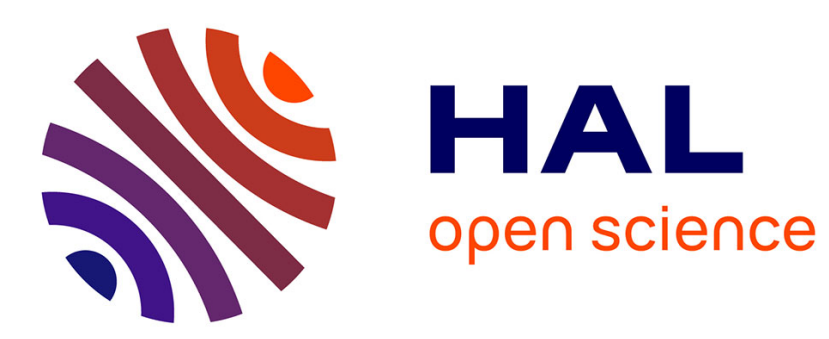

\title{
La tradition des textes sur Alexandre le Grand dans un proverbier glosé judéo-espagnol contemporain \\ Marie-Christine Bornes-Varol
}

\section{To cite this version:}

Marie-Christine Bornes-Varol. La tradition des textes sur Alexandre le Grand dans un proverbier glosé judéo-espagnol contemporain. Louchitsky S. \& Varol M.-C. Homo Legens - Styles and Practices of Reading: Comparative Analyses of Oral and Written Traditions in the Middle Ages, Brepols, pp.127-161, 2010, Utrecht Studies in Medieval Literacy, 9782503534091. hal-02139932

\section{HAL Id: hal-02139932 \\ https://hal-inalco.archives-ouvertes.fr/hal-02139932}

Submitted on 26 May 2019

HAL is a multi-disciplinary open access archive for the deposit and dissemination of scientific research documents, whether they are published or not. The documents may come from teaching and research institutions in France or abroad, or from public or private research centers.
L'archive ouverte pluridisciplinaire HAL, est destinée au dépôt et à la diffusion de documents scientifiques de niveau recherche, publiés ou non, émanant des établissements d'enseignement et de recherche français ou étrangers, des laboratoires publics ou privés. 


\section{La tradition des textes sur Alexandre le Grand dans un proverbier glosé judéo-espagnol contemporain}

\section{Introduction}

Entre l'écrit savant et l'oral populaire, les proverbes glosés, les coplas et les exempla occupent en Espagne au Moyen-Age (du moins pour les communautés juives où tous les hommes sont, par principe, des lecteurs-scripteurs) une place intermédiaire mi-religieuse, miséculière. Ce genre moral destiné à la prédication se transmet à la fois par l'oral et par l'écrit. Les grandes crises historiques que sont pour les Juifs d'Espagne l'expulsion de 1492, la crise religieuse de la fin du XVIIe siècle, et la shoah au XXe siècle ont à chaque fois entraîné des changements dans les modes de transmission de cette littérature tantôt écrite, tantôt orale. L'apparition de proverbiers glosés au XXe siècle et la nature des matériaux qu'ils contiennent comme leur agencement (les liens que ces proverbes entretiennent entre eux et avec des exempla) attestent de l'absence de solution de continuité dans la transmission d'un patrimoine parémiologique ancien. Dans cette perspective il nous a semblé intéressant d'étudier des éléments qui, selon toute vraisemblance, ont été acquis de manière ancienne et que l'on retrouve dans les traditions voisines (les textes juifs, arabes et chrétiens) de l'Espagne médiévale. Par souci de limiter le corpus nous avons choisi des énoncés proverbiaux figurant dans les textes relatifs à Alexandre ou rattachés à l'histoire d'Alexandre, au moyen âge en Espagne, et de regarder ce qu'il en restait dans un proverbier glosé judéo-espagnol contemporain. Pour ce faire nous avons d'une part parcouru une grande quantité de textes médiévaux sur Alexandre qui circulaient en Espagne et au-delà, relevé ce qui dans les textes en judéo-espagnol (après l'Expulsion des Juifs d'Espagne en 1492), était disponible ou accessible en matière de textes sur Alexandre. Enfin, nous avons repéré dans le proverbier les unités concernées. A partir du corpus de référence, nous avons soigneusement relevé les liens (ou l'absence de liens) entre ces énoncés, que l'auteur établissait dans ses gloses, ainsi que les liens qu'elle faisait avec d'autres énoncés proverbiaux ou exemplaires, puis nous avons considéré la nature des liens établis et des réseaux qu'ils constituaient. Retournant alors aux textes de départ, nous avons vu apparaître dans le proverbier des réseaux de sens sous-jacents organisant entre eux les proverbes et leurs gloses selon une logique reposant sur des textes écrits fondamentaux ou principaux, et en en éliminant d'autres. Nous analyserons ici en détail comment ces textes de référence organisent le discours au delà de la conscience qu'en ont leurs propres utilisateurs pour montrer que l'étude de «l'oral » est indissociable de l'étude de «l'écrit » (et vice versa) du moins dans la société judéo-espagnole.

\section{La «Clergie rabbinique » des Juifs d'Espagne}

\subsection{Les proverbiers glosés et les exempla dans la «clergie rabbinique » médiévale d'Espagne}

Entre l'écrit des textes sacrés et de leurs commentaires, les Juifs de l'Espagne au Moyen $\mathrm{Age}^{1}$ ont développé une littérature morale en langue vulgaire qui présente des corpus

\footnotetext{
${ }^{1}$ Les Juifs présents depuis l'époque romaine dans la Péninsule ibérique ont développé en Espagne araboandalouse une culture brillante souvent désignée comme «âge d'or des lettres hébraïques ». Au fur et à mesure de la reconquête chrétienne, ils ont formé des communautés de lecteurs - scripteurs et de lettrés dans les grandes villes de la Péninsule (en effet s'ils ne sont pas tous lettrés, tous les hommes juifs doivent au moins, par obligation religieuse, savoir lire et écrire). Ils ont été expulsés d'Espagne en 1492 et ont reconstitué des communautés dans les grandes villes du pourtour de la Mediterranée, principalement dans l'ex-Empire ottoman.
} 
de proverbes, sentences, maximes liés à des exempla, des fables, des contes et une poésie admonitive rimée dont les coplas qui forment un ensemble que Paloma Diaz Mas a dénommé la «clergie rabbinique $»^{2}$. Les ouvrages parémiologiques médiévaux qui contiennent les passages sur Aristote et Alexandre contiennent également de nombreux proverbes qui sont passés dans le proverbier judéo-espagnol et se sont transmis jusqu'à aujourd'hui. Enfin les Juifs d'Espagne connaissent aussi les ouvrages de proverbes glosés où les textes sentencieux sont mis en rapport avec des exempla. Ajoutons que cette tradition juive du musar (littérature morale ou admonitive) rejoint la littérature d'adab arabo-andalouse (d'où l'on tirait des normes morales et sociales de conduite). Dans le même souci d'instruire, elle mêle également les matériaux les plus «savants » aux matériaux les plus «populaires ».

Moshe Gaster a montré dans ces travaux ${ }^{3}$ que cette activité de compilation d'exempla prend son essor à partir du VIIIe siècle et se développe au XIe siècle (avec principalement l'Espagne arabo-andalouse pour centre) pour se poursuivre, après l'expulsion des Juifs d'Espagne, dans la diaspora séfarade où nombre de compilations sont imprimées. S'appuyant sur son incomparable ouvrage The exempla of the rabbis, A. Navarro et A. Alba, ont montré l'influence dans la littérature médiévale espagnole des recueils d'exempla rabbiniques constitués entre le VIII ${ }^{\mathrm{e}}$ et le $\mathrm{XI}^{\mathrm{e}}$ siècle comme le Midrash des dix Commandements, le Livre précieux de la Salvation (Hibbur Yafeh me Ha-Yeshu'a ou Sefer ha ma'asiyot) ${ }^{4}$ et les Alphabets de Ben Sira ${ }^{5}$, ou au $\mathrm{XIV}^{\mathrm{e}}$ siècle, comme le Livre des exemples des rabbins (également Sefer ha ma'asiyot), compilation faite en Espagne, qui compte trois-cents contes avec des ajouts et des emprunts de contes occidentaux ${ }^{6}$.

\subsection{Le sort de la « Clergie rabbinique » dans la diaspora}

Après l'Expulsion d'Espagne en 1492 les Juifs qui amènent l'imprimerie dans l'empire ottoman auront surtout à cœur d'imprimer en hébreu ou en langue-calque (ladino) les écrits sacrés, des codes de loi rabbiniques et quelques ouvrages de morale comme les Pirqe Avot ou 'Maximes des Pères', traité de la mishna réunissant aphorismes, commentaires et exempla. D'autres textes du même type feront l'objet de réeditions constantes : le texte tardif des Alphabets et de la Sagesse de Ben sira, recueils également d'aphorismes et exempla. Paraissent aussi des compilations de contes talmudiques comme Ein Yakov en hébreu et araméen.

Mais toute une partie de la tradition rabbinique semble interrompue avec l'expulsion d'Espagne. Les coplas paraliturgiques et admonitives disparaissent de l'écrit. Il en va de même des proverbes glosés et des exempla non talmudiques. Le courant de la «clergie rabbinique » semble tari et perdu.

Pourtant, après une grande crise religieuse survenue à la fin du XVII ${ }^{\mathrm{e}}$ siècle ${ }^{7}$, on voit brusquement réapparaître à l'écrit, en judéo-espagnol, des œuvres morales importantes : des

\footnotetext{
${ }^{2}$ Paloma Díaz-Mas, « Un Género casi perdido de la poesía castellana medieval : la clerecía rabínica », Separata del Boletín de la Real Academia Española, Tome 73 (259), Madrid, 1993.

${ }^{3}$ Moses Gaster, Judith Montefiore College Ramsgate. report for the Year 1894 - 1895 and Report for the Year 1895 - 1896 Together with The Ancient Collections of Agadoth The sefer ha-Maasiyoth and two Facsimiles, Ramsgate : Judith Montefiore College, 1896 / 5656 ; et The exempla of the Rabbis - being a collection of exempla apologues and tales, London: The Asia publishing Company, 1924.

${ }^{4}$ Amparo Alba Cecilia (ed.), 'Midrás de los Diez Mandamientos' y 'Libro precioso de la Salvación', Valence : Artes Gráficas Soler SA, 1990. (Biblioteca Midrásica 7. Institución San Jerónimo).

5 Elena Romero, Andanzas y prodigios de Ben-Sirá, Madrid : CSIC, 2001.

${ }^{6}$ Cf. à ce sujet Amparo Alba \& Angeles Navarro, «Del cuento rabínico al cuento medieval hispano » in Miscelánea des Estudios Arabes y Hebreos (section hébraïque) 53, 2004, pp. 17 - 34.

${ }^{7}$ La proclamation de Sabetay Sevi comme messie (en 1666) qui aboutit à la conversion à l'islam des disciples de Sabetay sous la pression du Sultan et à l'émergence de la secte des Dönme.
} 
coplas rabbiniques, qui seront imprimées pendant tout le $\mathrm{XVIII}^{\mathrm{e}}$ siècle ${ }^{8}$, ainsi que des commentaires rabbiniques de la Bible puisant abondamment dans les citations, les proverbes bibliques, et les exempla, talmudiques ou non. Les coplas qui sont des œuvres écrites et signées, sont imprimées mais se diffusent aussi oralement et nombre d'entre elles sont encore chantées de nos jours, du moins en partie. Le Me'am Lo'ez, commentaire rabbinique du Pentateuque que commence en $1732{ }^{9}$ le rabbin Ya'aqov Huli, compte citations bibliques, proverbes, sentences, contes et exempla, dont quelques textes sur Alexandre.

En dehors de cette transmission écrite, le proverbier, le contier et un énorme corpus de ballades bibliques, historiques ou lyriques chantées (les romances) font l'objet, pour leur part d'une transmission essentiellement orale, jusqu'à ce qu'une nouvelle rupture de la transmission, celle de la shoah au milieu du $X^{\mathrm{e}}$ siècle, produise de façon observable le passage à l'écrit d'une importante part de ce qui était transmis jusque-là oralement.

On peut néanmoins observer encore en synchronie (dans la deuxième moitié du $\mathrm{XX}^{\mathrm{e}}$ siècle) des pratiques de transmission orale, en situation, de corpus extrêmement codifiés tels que le proverbier glosé et les exempla. En Turquie, par exemple, où la communauté n'a pas été directement affectée par la coupure de la shoah, n'importe quelle situation de la vie quotidienne si elle est jugée porteuse de leçon et digne d'être transmise, appelle soit un proverbe déjà existant (et son commentaire ou / et un exemplum), soit est elle-même mise en forme rigoureusement, selon un moule textuel qui diffère nettement de la conversation ordinaire, pour produire un exemplum dont la morale ou la leçon peut être un proverbe ${ }^{10}$. Ou bien encore, à partir d'une situation nouvelle, un bon locuteur ou une bonne locutrice peut créer un «proverbe » (ou plutôt une formulation proverbiale ${ }^{11}$ ) sur des moules existant. Ce genre qui se situe entre l'écrit et l'oral (il s'agit en effet d'un oral très formalisé) sert à tenir des propos personnels médiatisés dans des situations où l'on ne peut directement exprimer son avis, et aussi à transmettre aux plus jeunes la sagesse du groupe augmentée de celle des parents, ou bien encore à mettre sous une forme transmissible des savoirs nouveaux jugés utiles au groupe.

Dans les communautés affectées par la shoah et par les grands mouvements de population du $\mathrm{XX}^{\mathrm{e}}$ siècle, la certitude de la fin d'un monde et d'une culture s'est imposée, si bien que l'on voit surgir à l'écrit des compilations de proverbes, glosés ou non, traduits ou

\footnotetext{
${ }^{8}$ Pour l'étude de ce genre en judéo-espagnol, on se reportera principalement à Elena Romero, Bibliografía analítica de ediciones de coplas sefardies, Madrid: CSIC, 1992 ; La creación literaria en lengua sefardí, Madrid: MAFPRE, 1992 (Chapitre IV); Seis coplas sefardies de castiguerio de Hayim Yom-Tob Magula, Madrid : CSIC, 2003 ; E. Romero \& Leonor Carracedo «Poesía judeoespañola admonitiva » in Sefarad, 37, 1977, pp. 429 - 451 ; et Paloma Diaz Mas, Temas y tópicos en la poesía luctuosa sefardí, Thèse présentée à Madrid, Université Complutense, 1982.

${ }^{9}$ Date de la parution en 1730 à Constantinople du premier tome (commentaire de Genèse); d'autres auteurs poursuivirent la tâche et commentèrent d'autres livres jusqu'à la fin du XIX $\mathrm{X}^{\mathrm{e}}$ siècle ( $c f$. à ce sujet E. Romero, $L a$ creación literaria..., op. cit., pp. 81 à 103). Seuls quatre ouvrages de cet ensemble ont fait l'objet d'une édition critique : Pascual Pascual Recuero \& David Gonzalo Maeso, Me'am Lo'ez - El gran comentario bíblico, Madrid : Gredos, (Prolegomènes), 1964 ; (Genèse, I \& II), 1969 \& 1970 ; (Esther), 1974 ; Rosa Asenjo, El Meam loez de Cantar de los Cantares (Šir haširim) de Hayim Y. Šaki (Constantinopla, 1899), Barcelone : Tirocinio, 2003 ; Aitor García Moreno, Relatos del pueblo ladinán (Me ám Lo 'eź de Éxodo), Madrid : CSIC, 2004.

${ }^{10}$ Cf. Marie-Christine Varol, «Les Judéo-Espagnols de Turquie et le discours indirect », in Hommage à Marthe Westphal, R. Caplán \& alii eds., Nancy: Institut d'espagnol et de portugais, 2001, pp. 151 à 179.

${ }^{11}$ Pour des raisons que j'ai expliquées ailleurs ( «Raíces medievales de los proverbios judeo-españoles » in Actes des Journées judéo-espagnoles de Londres, A. Benaïm ed., Londres : Queen Mary and Westfield college, 1999, pp. 203 à 218.), il n'est pas utile de distinguer pour le proverbier judéo-espagnol du Moyen Age (ni d'ailleurs contemporain) entre dit, dicton, sentence, proverbe, maxime, etc... J'emploie ici le terme 'proverbe' dans un sens très large.
} 
non, faits par les Judéo-Espagnols et publiés ${ }^{12}$. Parmi ceux-là, des proverbiers glosés mettant des proverbes en rapport avec des commentaires personnels, d'autres proverbes et des exempla, bien que partiels et très rares, sont apparus ${ }^{13}$. Ils sont destinés d'abord aux héritiers de ce judaïsme judéo-espagnol si spécifique et aujourd'hui en voie de disparition, puis aux Juifs en général et aux Autres enfin, pour communiquer une sagesse, mais ils ont aussi et surtout vocation de témoigner contre l'oubli et l'extinction d'une culture.

Le premier point qui nous intéresse ici c'est donc le passage à l'écrit d'une forme transmise oralement et la première observation étonnante est que la forme renoue, presque sans solution de continuité malgré le temps écoulé, avec une forme d'écrit médiéval.

\subsection{De l'oral à l'écrit : Le proverbier glosé de Mme Flore Gueron Yeschua ${ }^{14}$}

Mme Flore Gueron Yeschua est une dame cultivée de la bourgeoisie judéo-espagnole d'une petite ville bulgare (Tatar Pazardjik près de Plovdiv) qui est née en 1899. Elle a fait des études à l'école primaire juive puis au collège et au lycée bulgares et a voyagé en Europe où elle a fait quelques études à Rome, Vienne, Berlin et Genève. Elle n'a pas fait d'études religieuses. Elle a dû quitter la Bulgarie en 1943 avec son mari et ses fils pour se réfugier en Palestine. Elle a écrit ce proverbier dans les années 80 , à la mort de son mari et à la demande de son fils aîné Silvio. Il contient environ 800 proverbes (et expressions) commentés, qu'elle utilisait elle-même ou avait entendu utiliser et chacun est suivi d'une moyenne de six à sept lignes de commentaires en judéo-espagnol.

Le proverbier glosé de Mme Flore constitue donc un exemple rare en ce qu'il est une mise en écrit d'une tradition transmise oralement (en famille et en société), en contexte judéoespagnol, dans la diaspora séfarade de l'ex-Empire ottoman, et que nous disposons des précieuses gloses de l'auteur. En effet, par l'explicitation personnelle qu'elle fait du sens des proverbes et expressions qu'elle relève, par les renvois qu'elle fait d'un proverbe à l'autre, par les reprises de formulation à l'identique dans certains commentaires, par les liens qu'elle établit avec des exempla ou des anecdotes personnelles, Mme Flore nous permet d'accéder à l'architecture du proverbier comme corpus sapientiel partagé. Elle nous permet d'appréhender l'organisation sous-jacente à ces énoncés et les relations discrètes qu'ils entretiennent entre eux, pour l'une de leurs utilisatrices.

Dans le cadre du projet Homo Legens qui étudie les rapports entre écriture et oralité, j'ai recherché les traces d'Alexandre le Grand dans le proverbier de Mme Flore et décidé de rendre compte des liens éventuels, ou de l'absence de liens, faits par l'auteur entre ces énoncés et, le cas échéant, d'étudier la nature de ces liens. Le choix s'est porté sur Alexandre en raison de l'abondance des travaux critiques qui ont été consacrés aux chroniques, récits, romans, chansons et légendes sur ce héros et à leur circulation au Moyen age.

\section{La tradition espagnole d'Alexandre}

\footnotetext{
12 Cf., par exemple, Djamila Kolonomos, Poslovice, Izreke i Priče sefardskih jevreja makedonije - Proverbs, Sayings and Tales of the Sephardi Jews of Macedonia, Belgrade : Federation of the Jewish Communities in

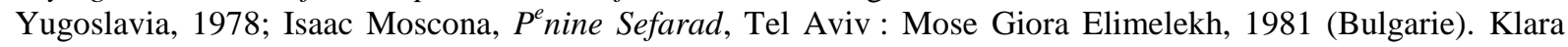
Perahya \& alii, Erensya Sefaradi, Istanbul : Gözlem, 1994 (Turquie). Enrique Saporta y Beja, Refranes de los judios sefardíes, Barcelona : Ameller, 1978 (Grèce).

${ }^{13} C f$. en dehors du proverbier manuscrit de Mme Flore qui doit faire l'objet d'une publication prochaine un petit livre du même ordre a paru récemment en Argentine : Luis León, Refranes y expresiones sefaradies - De la tradición judeo-española de Esmirna, Buenos Aires : Milá, 2001.

${ }^{14}$ Le manuscrit a fait l'objet d'une édition critique : M. C. Bornes -Varol, Le proverbier glosé de Mme Flore Gueron Yeshua (judéo-espagnol-Bulgarie), présentée dans le cadre de l'HDR (Paris IV-Sorbonne), qui doit être publiée prochainement aux éditions hispaniques.
} 


\subsection{Tradition hébraïque d'Alexandre en Espagne médiévale et dans la diaspora Judéo- Espagnole}

Ma recherche portant sur la postérité d'Alexandre dans le contexte judéo-espagnol, je me suis contentée d'un nombre raisonnable de textes pour mon corpus de référence.

En ce qui concerne l'héritage judéo-espagnol, j'ai considéré :

- les textes traditionnels anciens sur Alexandre contenus dans le Talmud et les midrashim;

- les textes ayant circulé dans l'Espagne arabo-andalouse et médiévale jusqu'à l'expulsion d'Espagne en 1492, en mettant l'accent sur ceux qui ont été traduits en hébreu au Moyen Age ;

- les passages sur Alexandre dans le Me'am Lo'ez de Genèse ${ }^{15}$;

- un opuscule publié en judéo-espagnol à Vienne à la fin du XIX ${ }^{\mathrm{e}}$, Historia de Alexandros el Mogdon rey de Makedonia qui reprend des morceaux des textes précédents ${ }^{16}$;

- les passages consacrés à Alexandre dans le Sefer Yosippon ${ }^{17}$; Les Exempla des Rabbins ${ }^{18}$.

Les textes que je présente sont ceux qui ont un rapport direct ou indirect avec le proverbier judéo-espagnol contemporain. Les deux épisodes talmudiques qui se trouvent en jeu dans le proverbier de Mme Flore sont «Alexandre et les sages du sud» et «Alexandre à la porte du jardin d'Eden ». Ils sont repris dans le Me'am lo'ez de 1730, figurent dans l'opuscule de Vienne et dans un conte ayant Alexandre pour héros, recueilli par la folkloriste Matilda Koen Sarano. Enfin on trouve le passage concernant le banquet qu'Alexandre commande à Olympias pour la consoler de sa mort prochaine, utilisé comme exemplum dans le texte judéo-espagnol du traité de morale Pele Yo'ets, de Eliezer Papo, à la fin du XIX ${ }^{\mathrm{e}}$ siècle ${ }^{19}$.

\subsection{Les textes partagés en Espagne médiévale}

J'ai examiné principalement les textes du Pseudo-Callisthène qui est au Moyen Age un ouvrage très diffusé.

- Le premier groupe de textes examinés sont des traductions du Pseudo-Callisthène et du texte latin De Proeliis ${ }^{20}$.

- La version occidentale en arabe qui serait issue d'une version syriaque du PseudoCallisthène ${ }^{21}$ éditée et traduite de l'arabe en espagnol par E. Garcia Gomez et un texte

\footnotetext{
${ }^{15}$ P. Pascual Recuero \& D. Gonzalo Maeso, Me'am Lo'ez... (Genèse I) (op. cit.), pp. 53 à 57.

${ }^{16}$ Historia de Alexandros el Grande rey de Makedonia - Lectura muy interessante y provechosa, Vienne : Joseph Schlesinger, 1890 (en caractères rashi).

${ }^{17}$ L'édition en judéo-espagnol de Salonique (1863) et une édition critique moderne de Moshe Lazar, Sefer Ben Gurion [Yosipon] - First Ladino Translation by Abraham Asa [1753], Lancaster (Cal.) : Labyrinthos, 2000.

${ }^{18}$ M. Gaster, The Exempla of the Rabbis..., (op. cit.).

${ }^{19}$ Eliezer Papo, Pele Yo'ets, Vienne : Jacob Hacohen Shlosberg, 1870 (tomeI) et 1872 (II) (en caractères rashi)

${ }^{20}$ Pseudo-Callisthène, Le roman d'Alexandre - La vie et les Hauts faits d'Alexandre de Macédoine, Traduit et commenté par G. Bounoure et B. Serret, Paris : Les belles-Lettres, 1992 ; Le roman d'Alexandre, trad. Aline Tallet Bonvalot, Paris : Garnier Flammarion, 1994. Pour l'histoire des textes voir aussi George Cary, The medieval Alexander, Cambridge : Cambridge University Press, 1956 ; Laurence Harf-Lancner \& alii (eds) Alexandre le Grand dans les littérature occidentales et proche-orientales - Actes du colloque de Paris 27 - 29 novembre 1997, Université de Paris X- Nanterre, 1999 ( Littérales hors série 199) ; Corinne Jouanno, Naissances et métamorphoses du Roman d'Alexandre (Domaine grec), Paris : CNRS, 2002.

${ }^{21}$ Faustina C. W. Douficar - Aerts, « Les derniers jours d'Alexandre dans un roman populaire arabe : un miroir du roman syriaque du Pseudo-Callisthène » in L. Harf-Lancner \& alii, Alexandre le Grand... (op. cit.), p. 61.
} 
aljamiado morisco de la légende d'Alexandre qui se sont révélés, comme on le verra, particulièrement intéressants 22 .

- Les traductions de De Proeliis en hébreu :

- celle qui est attribuée à Ibn Tibbon $(1150$ - 1230) est peut-être issue d'une version antérieure; elle offre des ressemblances avec certaines parties insérées dans le sefer Yosipon, daté de la fin du $\mathrm{X}^{\mathrm{e}}$ siècle ;

- le texte d'une autre version hébraïque plus fantaisiste selon I. Lévi, plus proche des sources orales et des légendes populaires sur Alexandre d'après M. Gaster ${ }^{23}$;

- Le Sefer Toledot Alexandros ha Makdoni, traduction de Immanuel Bonfilio de Tarascon au milieu du $\mathrm{XIV}^{\mathrm{e}}$ siècle $^{24}$, qui ajoute à De Proeliis des passages issus du Mussre ha Pilosophim et de Sod ha sodot (cf. infra).

L'épisode des Gymnosophistes ou des dix questions aux sages de l'Inde qui circulait indépendamment au Moyen $\mathrm{Age}^{25}$, et qui est présent dans les textes judéo-espagnols, a été ensuite confronté dans un nombre plus large de versions orientales et occidentales du PseudoCallisthène, et rapproché de celle de Plutarque ${ }^{26}$.

Un second groupe de textes examiné est directement issu de la tradition arabe sur Alexandre :

- Le Pseudo-Aristote, Sir al-Asrar en arabe et la traduction espagnole de sa version occidentale Poridat de las poridades ${ }^{27}$. Une traduction hébraïque Sod ha sodot, attribuée à al-Harizi (XIII ${ }^{\mathrm{e}} \mathrm{s}$.). Le thème en est un traité de gouvernement de l'Inde, rédigé par Aristote à la demande d'Alexandre.

- Le Kitab adab al-falasifa ${ }^{28}$, traduction et compilation de sentences, maximes et exempla du grec par le traducteur syriaque Honayn Ibn Ishak $\left(\mathrm{IX}^{\circ} \mathrm{s}\right)$ qui a donné deux autres œuvres postérieures en arabe qui circulaient en Espagne. Le livre éponyme de Muhammad al-Ansari ( $\mathrm{XI}^{\mathrm{e}}$ s.) et Muhtar al-Hikam wa Mahasim al-Kalim, (achevé en 1053) de Mubashshir ibn Fatik ${ }^{29}$. Le premier, traduit en espagnol sous le nom de Libro de los Buenos Proverbios a eu une diffusion limitée à l'Espagne dans les langues de la Péninsule mais a connu une diffusion plus large dans le monde juif grâce à sa

\footnotetext{
${ }^{22}$ Guillén Robles, Leyendas de José Hijo de jacob y de Alejandro magno sacada de dos manuscritos Moriscos de la biblioteca nacional de Madrid, Zaragoza : Imprenta del Hospicio, 1888 ; Emilio García Gomez, Un texto arabe occidental de la leyenda de Alejandro, Madrid : Instituto de Valencia de Don Juan, 1929 ;

${ }^{23}$ Ce texte qui se trouve dans trois manuscrits (Modène, Bodleian et Damas) a été édité et traduit par Moses Gaster, An old romance of Alexander (1897) et reproduit dans Studies and Texts in folklore, Magic,... (op. cit.) Vol. II, p. 814.

${ }^{24}$ The Book of the Gest of Alexander of Macedon - Sefer Toledot Alexandros ha Makdoni / a Medieval Hebrew Version of the Alexander Romance by Immanuel Ben Jacob Bonfils, Israel J. Kazis (ed.), Cambridge Mass: The medieval academy of America, 1962. Pour la comparaison des textes juifs sur Alexandre on se reportera aux articles suivants de Israël Levi, parus dans la Revue des Etudes Juives : «La légende d'Alexandre dans le Talmud », II, 1881, pp. 293 à 300 ; «Les traductions hébraïques de l'histoire légendaire d'Alexandre », III, 1881, pp. 238 à 265 ; «La Légende d'Alexandre dans le Talmud et le Midrasch », VII, 1883, pp. 78 à 93.

${ }^{25}$ Cf. Corinne Jouanno op. cit., pp.XX

${ }^{26}$ Plutarque, Vie d'Alexandre in Vies Parallèles, Tome I, Paris : GF Flammarion, 1995.

${ }^{27}$ Pseudo Aristote, Poridat de las poridades, Lloyd Kasten ed., Madrid : Silverio Aguirre, 1957 ; Charles B. Schmitt (eds), Pseudo-Aristotle The Secret of Secrets Sources and Influences (Warburg Institute Surveys IX), Londres : University of London, 1982.

${ }^{28}$ Pour l'étude critique de ce texte et sa circulation on se reportera principalement à Dimitri Gutas, Greek Wisdom literature in arabic translation : a study of the Graeco-Arabic Gnomologia, New Haven : American Oriental Series, vol. 60, 1975. Cf. également: Manuel Alonso Alonso, « Dawud y Domingo Gundisalvo » in Al-Andalus, 16, 1951, pp. 37 à 48 ; John K. Walsh, «Versiones peninsulares

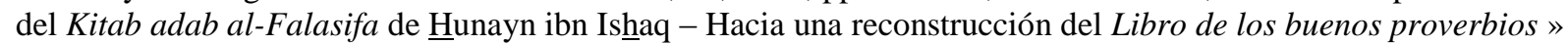
in Al-Andalus, 41 (2), 1976, pp. 355 à 384.

${ }^{29}$ Sur le texte arabe, sa circulation et ses liens avec le Kitab adab al-Falasifa, voir Franz Rosenthal, « AlMubashshir ibn Fâtik, Prolegomena to an abortive edition » in Oriens, 13 - 14, 1960 - 61, pp. 132 - 158.
} 
traduction en Hébreu sous le nom de Mussre ha-Philosophim par Yehudah al-Harizi ${ }^{30}$. Le second n'a pas été traduit en hébreu, mais sa traduction en espagnol sous le nom de Bocados de oro a donné lieu à une traduction latine Bonium qui s'est considérablement diffusée en Europe. Une partie du matériel est commune à Bocados de oro et à Buenos Proverbios ${ }^{31}$. On retrouve un fragment commun dans la Disciplina Clericalis de Pedro Alfonso de Huesca (début du XII ${ }^{\mathrm{e}}$ s.) : celui des sentences des sages groupés autour du tombeau d'or d'Alexandre ${ }^{32}$. Les énoncés tirés de ces sommes se retrouvent aussi dans des textes comme llibr de dits e proverbis de savis e filosofs de Yehudah Bonsenyor ( $\operatorname{circa} 1260)^{33}$, ainsi que dans le livre d'aphorismes et exempla attribué à Ibn Gabirol, d'abord paru en arabe mais dont on n'a conservé que la version hébraïque traduite Mivhar ha-P $P^{e}$ ninim $^{34}$.

Comme on l'a dit, les textes de ces différents ouvrages se confondent ou se recoupent souvent ${ }^{35}$, particulièrement les passages portant sur les derniers jours et la mort d'Alexandre ${ }^{36}$.

\section{Les proverbes d'Alexandre dans le proverbier glosé}

\subsection{Le corpus étudié}

Que reste-t-il d'Alexandre dans le proverbier judéo-espagnol glosé de Mme Flore ? Il n'apparaît pas directement. On ne cite pas le nom d'Alexandre (en général il ne figure pas dans le proverbier judéo-espagnol) ni dans les gloses de Mme Flore. Il pourrait apparaître comme « le roi, un roi... » mais on ne trouve rien de probant dans ce sens. Par contre deux épisodes contenus dans les gloses et plusieurs énoncés proverbiaux sont identiques à ceux figurant dans les textes médiévaux ou les récits légendaires sur Alexandre, dans un nombre important de corpus, dont les corpus proprement juifs (Talmud et commentaires rabbiniques).

J'ai relevé les cinq unités suivantes ${ }^{37}$, et un sixième proverbe qui offre une formulation proche et qui dérive des mêmes corpus sans coïncider à la lettre. Ils ne constituent pas les seuls énoncés en rapport avec Alexandre : une autre unité, par exemple, Dinguno no fuye de su destino, 'Nul n'échappe à son destin', si elle figure dans un corpus sur Alexandre (l'épisode de la mort de Nectanebo), est trop largement diffusée au moyen Age pour être considérée comme propre aux corpus sur Alexandre. Le proverbier judéo-espagnol compte d'autres unités figurant souvent dans les corpus sur Alexandre tels que El enemigo, faztelo

\footnotetext{
${ }^{30}$ Le texte de De Proeliis traduit par Emmanuel Bonfilio contient des fragments du Mussre ha philosophim et de Sod ha-sodot (I. Kazis, $1962: 1$ ).

${ }^{31}$ Les versions espagnoles des deux textes ont été éditées et comparées par Hermann Knust, Libro de los buenos proverbios que dixieron los phi(losophos) in Mittheilungen aus dem Eskurial, Bibliothek des litterarischen Verein, 141, Tubingen, 1879. Pour le texte de Libro de los Buenos Proverbios nous nous sommes appuyée sur l'édition de H. Sturm, The «Libro de los buenos proverbios » - A Critical Edition, The University Press of Kentucky, 1971. Pour le texte de Bocados de Oro, sur l'édition de Mechtild Crombach, Bocados de Oro, Bonn : Romanischen Seminar des Universitat, 1971.

${ }^{32}$ Angel Gonzalez Palencia (éd.), 'Disciplina Clericalis' - Edición y traducción del texto latino, Madrid : CSIC, 1948.

${ }^{33}$ Jafudá Bonsenyor, Libro de palabras y dichos de sabios y filósofos, édition et traduction de J. R. Magdalena Nom de Deu, Barcelona : Riopedras, 1990.

${ }^{34}$ Shelomó ibn Gabirol, Selección de perlas - Mibhar ha-peninim, édition et traduction de D. Gonzalo Maeso, Barcelone : Ameller, 1977. Sur l'attribution à Ibn Gabirol voir Yehudah Ratzhabi, « Les sources arabes du Mivhar ha-p ninim » in Sinä̈, n 102, Jérusalem, 1988, pp. 97 à 160 (en hébreu).

${ }^{35} C \bar{f}$. à ce sujet notamment Georges Cary, op. cit.

${ }^{36}$ W. J. Aerts \& alii (eds), Alexander the great in the Middle Ages :Ten studies on the last day of Alexander, Nimègue : Alfa, 1978 et Faustina Douficar Aerts in L. Harf-Lancner \& alii (ed.), article cité note 21.

${ }^{37}$ Les lettres et les chiffres entre parenthèses, (E47), renvoient à la numération du proverbier de Mme Flore. Les chiffres en gras, 6 , ont été attribués pour cette étude aux proverbes principaux figurant dans les tableaux.
} 
amigo, 'ton ennemi, fais-t-en un ami' (corpus du Pseudo -Aristote), mais, comme ils ne figurent pas dans le proverbier de Mme Flore, ils ont été écartés de cette étude.

1- $\quad$ Si no vyene la ora del Dyo una oja no kaye del arvolé (S45)

'Si l'heure de Dieu ne vient pas, une feuille ne tombe pas de l'arbre'

2- $\quad$ Kén es el riko ? El ke se kontenta kon su parte (K52)

'Qui est le riche? Celui qui se contente de sa part'

3- $\quad$ El ojo del bivo se arta solo kon tyera (E47)

'L'œil de [celui qui est] vivant ne se rassasie qu'avec de la terre'

4- $\quad$ Se arastó lo seko kon lo vedre (S29)

'Le sec a été arraché avec le vert'

5- $\quad$ Las dadas son tomadas (L50)

'les [choses] données sont prises'

et

6- $\quad$ Dinguno no muere si el Dyo no lo kere (D15)

Personne ne meurt si Dieu ne le veut

\section{2 Les relations entre les unités : méthode et résultats}

\section{2. 1 Présentation des tableaux 1 et 2}

Les énoncés sélectionnées ont été rapportés dans le tableau 1, écrits en gras, encadrés par des rectangles et numérotés en gras.

Chacun de ces énoncés proverbiaux est directement relié par Mme Flore à des proverbes ou des expressions figurant par ailleurs comme entrées dans son proverbier, écrits en noir et également encadrés par des rectangles.

J'ai ensuite établi les réseaux complexes de relations que ces unités entretenaient avec d'autres, à partir

$1^{\circ}$ ) des citations et des renvois de proverbe à proverbes, établis explicitement par Mme Flore, qui sont signalés par des traits droits reliés à des pastilles rondes ;

$2^{\circ}$ ) des commentaires partagés par différents proverbes, figurant dans leurs gloses. Ces énoncés sont inscrits en clair dans des bulles ovales reliées entre elles par des traits courbes ou sinueux.

Ces informations ont été portées sur le tableau 2.

En examinant les tableaux 1 et 2 on voit que les recoupements opérés par Mme Flore regroupent les «énoncés d'Alexandre » en trois parties ou trois groupes de deux: les proverbes $\mathbf{3}$ et $\mathbf{2}$ à droite du tableau; les proverbes $\mathbf{1}$ et $\mathbf{6}$ en haut à gauche du tableau; les proverbes 4 et 5 en bas à gauche du tableau. Alors que les énoncés 1, 2, 3, 6 entretiennent un grand nombre de liens, 4 \& $\mathbf{5}$ apparaissent sans liens entre eux ni avec le reste des énoncés.

3. 2. 2 Une métaphore centrale : L'oeil insatiable de l'homme

Les deux premières unités, en haut à droite, 3- El ojo del bivo... (E47) et 2- Ken es el riko ?... (K52) dessinent un réseau serré d'énoncés sentencieux ou proverbes nombreux, reliés entre eux essentiellement par leurs commentaires situés dans les ellipses. 
Ils tournent d'abord autour du thème du désir et de l'insatisfaction humaine, représentés par un œil vide qui ne se satisfait que de la terre du tombeau (qui n'est rassasié que par la mort). La métaphore centrale est illustrée par un exemplum raconté in extenso dans la glose du proverbe E47 par Mme Flore ${ }^{38}$. Ensuite, ils évoquent la nécessité pour l'homme de se contenter de son sort sans chercher à obtenir davantage : (K52) Kén es el riko... 'Qui est le riche ? Celui qui se contente de sa part / de ce qu'il a'.

On remarquera que, dans les commentaires, on désapprouve ceux qui se lancent dans des aventures et ceux qui veulent obtenir le monde entier et ses richesses, traits qui se rapportent ordinairement à Alexandre, qui se voit souvent reprocher la vanité de son ambition (par différents messagers des peuples qu'il soumet, par les Gymnosophistes, par les philosophes réunis autour de son tombeau...). De façon logique, par les gloses, se trouve lié à cet ensemble un enseignement sur l'envie qui cause l'usure et la mort :

(E67) el selo i el dezeo kitan a la persona del mundo 'la jalousie et le désir ôtent la personne du monde'.

Apparaît également, ouvert par la métaphore de l'œil béant, un lien entre le regard et le désir : (V3) Ver i dezear, 'Voir et désirer'. Ce dernier a le sens exact de 'Voir [c'est] désirer', puisqu'il est relié au proverbe (K32) Ken mira la djente no bive kontente, 'Qui regarde [ce qu'ont] les gens, n'est jamais content', par l'énoncé commun à leurs deux gloses : en mirando los byenes d'enfrente no ay repozo para dinguno, littéralement 'quand on regarde les biens d'en face, il n'y a de repos pour personne'.

Mme Flore lie par ses enseignements trois autres proverbes à cet ensemble, alors qu'ils présentent pourtant des idées différentes. On les trouvera sur le bord droit du tableau.

Il s'agit d'abord, tout en haut à droite, de (T31) Todo vyene de la tyera $i$ se va a la tyera, 'Tout vient de la terre et retourne à la terre' ou 'Tu es poussière et tu redeviendras poussière'. Le commentaire lie l'insatisfaction (son muy pokos los ke se kontentan kon lo ke tyenen, 'ils sont rares ceux qui se contentent de ce qu'ils ont') à la vanité du monde qui n'aboutit qu'à la mort (la terre du tombeau).

Le second proverbe relié à cet ensemble est, sur le bord droit du tableau un peu plus bas, présente un passage de l'abstrait au concret, de l'œil vide au gosier vide : (K43) Ken tyene el ojo vaziyo no tyene nunka el papo entchido, 'Qui a l'œil vide n'a jamais le gosier rempli'.

Le troisième enfin, en bas à droite, (K32) Ken mira la djente no bive kontente, 'Qui se soucie des gens (ou regarde les gens) ne vit jamais content', dont la leçon, liée à l'exemplum «Le meunier, son fils et l'âne », est ordinairement «bien faire et laisser dire » ${ }^{39}$. En raison du vocabulaire utilisé (regarder et se contenter), il se voit adjoindre ici une nouvelle leçon très différente, celle de ne pas convoiter ce que les autres ont : En mirando los byenes d'enfrente no ay repozo para dinguno 'si chacun regarde les biens d'autrui, il n'y a de repos pour personne'.

\footnotetext{
${ }^{38}$ Le récit, traduit du judéo-espagnol est le suivant: 'On raconte une petite histoire où il est question d'un ouvrier qui trouva dans un trou un petit os. Il le lava, le nettoya et voulut savoir combien il pesait. Il le mit sur la balance et commença à poser les poids sur l'autre plateau. Il en mit tant et plus et rien ne suffit pour estimer son poids. Vint alors une voisine âgée qui vit les soucis du jeune homme. Dès qu'elle vit le petit os, elle poussa un cri et lui dit "Enlève tous ces poids et remplace-les par un peu de terre". A peine l'eut-il fait que la balance pencha de ce côté. "Ce petit os - dit la femme - provient du côté d'un oeil humain, tu ne peux le rassasier qu'avec de la terre".

${ }^{39}$ Cf. Reginetta Haboucha, Types and Motifs of the Judeo-Spanish Folktales, New York : Garland, 1992, p. 525 AT 1215 The Miller, His Son and the Ass: Trying to please everyone; Ysopete J1041.1; Tubach Index Exemplorum, $\mathrm{n}^{\circ}$ 382; et p. 527 pour les sources médiévales arabes et espagnoles. Matilda Koen Sarano, Kuentos del folklore de la famiya djudeo-espanyola, Jérusalem : Kana, 1986, p. 93, l'exemplum a le proverbe Ken mira la djente no bive kontente, pour titre. Sur la circulation médiévale de cet exemplum dans les textes espagnols, $c f$. Daniel Devoto, Introducción al estudio de Don Juan Manuel, y en particular de El Conde Lucanor - Una bibliografia, Paris : Ediciones Hispano-Americanas, 1972, p. 364.
} 
Les autres sentences qui sont liées par les commentaires aux deux autres ont, elles un lien thématique proche et logique avec les deux énoncés centraux. (K1) Ken byen está $i$ mejor eskoje, kuando le vyene el mal ke no se anoje, 'Qui se trouve bien et choisit mieux, qu'il ne s'en prenne à personne s'il lui advient du mal', situé tout en haut du tableau vers le milieu, est directement relié au proverbe (juste en dessous) (K27) Ken mas tyene mas kere, 'qui a plus veut plus'. Ce dernier est repris sous une autre forme dans la glose tyenen i tyenen i mas keren 'plus ils en ont, plus ils en veulent'. Il est indirectement lié à (N17) No dezees lo ke alkansar no puedes, 'ne désire pas ce que tu ne peux atteindre' par le commentaire déjà cité 'rares sont ceux qui se contentent de ce qu'ils ont' et à (K40) Ken kere lo mutcho pyedre $i$ lo poko 'qui veut beaucoup ( ou trop) perd le peu qu'il a', par le commentaire commun sur les aventuriers qui 'en quête de grandes richesses se lancent dans les aventures et perdent le peu qu'ils avaient', dezean alkansar rikezas grandes, se etchan en avanturas $i$ pyedren lo poko ke tyenen. (N17) No dezees... est lui-même lié à un commentaire qui le reformule Se deven kontentar kon lo ke pueden alkansar, 'Les hommes doivent se contenter de ce qu'ils peuvent obtenir'.

Le commentaire sur les aventuriers est relié logiquement au commentaire sur le désir insatiable de 'la personne dont l'œil vide ne se rassasie pas de ce qu'il a', Persona ke tyene el ojo vaziyo no se konsyente arto de lo ke tyene. Il est également relié à deux autres commentaires qui contiennent aussi la référence au désir d'obtenir davantage (dezean alkansar) : Los selozos dezean alkansar el mundo entero, los otros se kontentan kon lo ke tyenen, 'les envieux désirent obtenir le monde tout entier (rappelons que l'exemple d'Alexandre est à ce sujet prototypique), les autres se contentent de ce qu'ils ont' et No syempre la persona puede alkansar lo ke dezea en la vida // No prime enselado del byen ke tyene el d'enfrente, 'L'homme ne peut pas toujours obtenir ce qu'il désire dans la vie // Il ne convient pas d'être jaloux du bien de son prochain'. Ce dernier commentaire est lié avec (K52) Ken es el riko ?... 'Qui est le riche ?...', (E76) El selo i el dezeo... 'la jalousie et le désir ou la convoitise...', et (V4) Ver i dezear, 'voir et désirer'.

On a donc un réseau étroit de commentaires moraux de type eux-même sentencieux qui forment la leçon des proverbes sur le désir insatiable des hommes qui les porte à ne pas se contenter de ce qu'ils ont, à jalouser le bien de leur prochain, à perdre le repos et à périr, rongés par l'envie ; à chercher à obtenir plus qu'ils ne peuvent atteindre en se lançant pour cela dans des aventures et, en définitive, à finir par tout perdre.

Trois énoncés thématiquement plus éloignés se trouvent rattachés à cet ensemble cohérent, l'un sur la mort inéluctable symbolisée par la terre du tombeau (T31), l'autre sur une variante plus concrète qui lie l'envie (insatisfaite) à la faim (insatisfaite) et au gosier vide (K43), et enfin un proverbe qui a d'ordinaire une autre leçon qui semble glisser ici de 'suivre l'opinion des gens' à 'convoiter le bien des gens' (K32), ce qu'il va falloir expliquer.

\section{2. 3 La célébration de la toute-puissance divine}

Un deuxième groupe de sentences d'Alexandre, qui contiennent le terme Dieu ou la mention de l'une de ses désignations, est formé par Mme Flore, principalement par le biais de renvois de proverbe à proverbe. Il s'agit en l'occurrence de nombreuses bénédictions ou formules religieuses, qui portent sur la toute-puissance de Dieu et la nécessité de lui rendre grâce quel que soit le sort qui nous est réservé. Ils sont situés en haut et à gauche des tableaux 1 et 3. Le proverbe 1 d'Alexandre (S45) Si no vyene la ora del dyo... et sa leçon formulée dans le proverbe 6 dont la formulation est proche (D15) Dinguno no muere si el Dyo no lo kere, 'Personne ne meurt si le Dieu ne le veut', renvoient aux proverbes (D15) Orozos son los kreyentes, 'Heureux sont les croyants' (proverbe bulgare), à la formule de bénédiction (B 3) Benditcho el ke le plaze, 'Béni soit ce qui Lui plaît'; au souhait (E67) El Dyo ke no dé 'Que 
Dieu ne nous donne pas (sous-entendu cette épreuve)'ou 'Puisse Dieu nous en préserver', courant lorsqu'il est question de la mort. Le même énoncé (D15) Dinguno no muere... est également directement lié au proverbe qui compare la nécessité de se résigner à la volonté divine à l'exemple de la docilité de la terre qui reçoit les intempéries du ciel (K51) Ké dan de los syelos ke no resive la tyera?, 'Qu'est-ce que le ciel envoie que refuse la terre ?'. Le proverbe (D15) Orozos son los kreyentes (qui vient du bulgare) est pour sa part directement lié (toujours par les renvois et citations explicites de proverbe à proverbe faits par Mme Flore) à (K51) Ké dan de los syelos... et à (E22) El Dyo ke no mos apreve, 'Que Dieu ne nous mette pas à l'épreuve' qui est cité par Mme Flore sous le même numéro que (E22) A los buenos apreva el Dyo, 'Les gens de bien, Dieu les met à l'épreuve'. Comme nous le verrons infra, Mme Flore cite entre parenthèses dans son commentaire la référence à Job.

Les proverbes qui ne sont pas reliés à d'autres, sont liés par des commentaires dans lesquels figurent les multiples noms donnés à Dieu, qui témoignent de sa toute puissance contre laquelle il est vain de s'élever : (E67) El Todo Poderoso 'le Tout Puissant', El Patrón del mundo, 'le Maître du monde" 40 .

Ils sont également liés par des commentaires sur la consolation, l'espérance et la patience qu'offre la foi : La kreensa en el Dyo es muy grande, se konsolan los kreyentes, 'La croyance en Dieu est grande, les croyants sont consolés' ; Esperansa en el Dyo, 'l'espérance (est) en Dieu ; (los kreyentes) echan los ojos al syelo, '(les croyants) lèvent leurs yeux vers le ciel' ; pasensya i konsolasyón 'patience et consolation', expression qui sert à consoler les gens affligés.

Ils sont liés, enfin, par des commentaires portant sur l'impénétrabilité et l'implacabilité des lois du destin : Etchas del Dyo, 'faits (décidés) par Dieu'; es etchado de los syelos, 'c'est décidé (jeté) par les Cieux'. L'exemple auquel ces énoncés se trouvent rapportés explicitement est celui de Job et ils sont également liés, par les gloses, au texte de la prière pour les morts ou kaddish dont la première phrase est citée en hébreu.

Le dernier groupe de commentaires (situé dans la bulle la plus à gauche et en bas du tableau 1) est relié directement à un autre proverbe (P11) Para maraviyas del Dyo no ay kavo, 'il n'y a pas de limite aux merveilles de Dieu', qui tranche par son optimisme avec la thématique dominante de cette partie, à savoir la résignation nécessaire à la volonté divine dans ce qu'elle a de plus rigoureux et la crainte des épreuves et de la mort. Il est la seule sentence de cet ensemble à fonder l'espérance envers et contre tout. Il exprime la confiance en un Dieu bienveillant et prodigue et semble isolé dans cet ensemble pessimiste auquel ne le rattache que la proclamation de la toute-puissance divine, miraculeuse et infinie.

\section{2. 4 Entre le désir vain et la toute-puissance divine : le problème de la rétribution}

Entre le groupe des énoncés qui est à droite (et qui porte sur le désir insatiable et vain qui épuise l'homme jusqu'à sa mort) et celui de gauche (sur la nécessité de se soumettre à la volonté d'un Dieu rigoureux), il n'y a qu'un seul lien direct, ténu, formé par la glose se deven kontentar kon lo ke pueden alkansar 'ils doivent se contenter de ce qu'ils peuvent obtenir', commune entre (P24) Por lo k'estamos, bindigamos! 'Rendons grâces à Dieu pour notre état' et (N17) No dezees lo ke alkansar no puedes, 'Ne désire pas ce que tu ne peux atteindre'.

Par contre on constate que tant l'ensemble de droite que celui de gauche sont étroitement liés par les commentaires et les renvois à un ensemble serré de proverbes qui sont par leur forme et leur sens assez éloignés des deux autres groupes, mais qui portent tous sur

\footnotetext{
${ }^{40}$ De façon assez significatives ces dénominations, comme les bénédictions et souhaits qui figurent dans ce tableau constituent des entrées du proverbier glosé de Mme Flore. Je les inclus, comme elle le fait elle-même, sous le titre générique de «proverbes » (cf. supra note 11).
} 
les voies impénétrables de la Providence et la question de la rétribution, en ce monde, des justes et des méchants :

- Le proverbe (A8) Al riko i el gayo le mete guevo, al prove, ni la gayna, 'Le riche même son coq lui pond un oeuf, le pauvre, pas même sa poule' qui porte sur l'injustice du sort qui s'acharne sur les plus pauvres et favorise toujours les mêmes.

- La sentence en hébreu, suivie de sa traduction en judéo-espagnol : El bueno tyene lo negro i el negro tyene lo bueno, 'Le bon a la mauvaise part et le méchant a la bonne part', qui figure sous le proverbe (E25)(cf. infra) comme ayant le même sens que lui.

- Le proverbe (E25) El dyo da barvas al ke no tyene kechadas, 'Dieu donne de la barbe à qui n'a pas de mâchoires', que l'on connaît aussi sous la forme 'Dieu donne du pain à celui qui n'a pas de dents'. Il déplore le manque de discernement du sort, ou son ironie malveillante.

Il faudra expliquer ce lien qui n'apparait pas comme immédiatement logique, et dont la cohérence ne repose pas sur l'histoire d'Alexandre.

\section{2. 5 Deux sentences à part (4 et $\mathbf{5})$ sur les aléas de la Providence}

Un troisième groupe de sentences, 4, (S29) Se arasto lo seko kon lo vedre, 'le vert a été arraché avec le sec', et 5, (L50) Las dadas son tomadas, 'Les choses données sont reprises /ou rendues', réunit les proverbes d'Alexandre qui ne sont pas reliés aux autres. Ceci présente une énigme, car dans les corpus de proverbes espagnols médiévaux (S29) 'Le sec a été arraché avec le vert' est relié à (P2) Pagan djustos por pecadores, 'Les justes payent pour les pécheurs' qui porte aussi sur la question de la rétribution ${ }^{41}$. Mais ces énoncés (S29) et (L50), font partie chez Mme Flore de tout autres domaines d'explicitation et réseaux de proverbes. Le proverbe (P2) qui figure dans son proverbier n'est pour sa part relié, ni par renvoi direct ou citation, ni par le biais des commentaires, aux autres proverbes figurant sur les tableaux 1 et 3 . Le commentaire de Mme Flore à $(\mathrm{P} 2)$ donne des exemples concrets, tirés d'épisodes de la vie courante, sans aucune référence aux voies de la Providence ou à la justice divine.

(L50) Las dadas son tomadas, reçoit une explication particulière qui lui donne un sens différent de sa traduction / interprétation dans les corpus d'Alexandre, à savoir 'on reçoit ce que l'on a donné', que Mme Flore met d'ailleurs en rapport avec une autre formulation proverbiale: Lo ke azes, akeyo aresives, 'on agit avec toi comme tu agis toi-même' qui signifie que l'on récolte ce que l'on sème.

(S29) reçoit une explication portant sur le sens propre qui en fait une simple expression signifiant que tous les recours, ou toutes les provisions ont été épuisés ou bien que tout ce qui était à dire a été dit.

\section{La confrontation du proverbier avec les textes médiévaux sur Alexandre}

\subsection{La confrontation croisée des textes sur Alexandre et des corpus proverbiaux}

Qu'est-ce qui fait que certains énoncés sont liés, et de cette façon, tandis que d'autres sont écartés ? Pourquoi les deux ensembles reliés, le sont par l'intermédiaire d'un troisième, a

\footnotetext{
${ }^{41}$ Cf. Eleanor O'Kane, Refranes y frases proverbiales españolas de la Edad Media, Anejos del Boletín de la Real Academia Española (II), Madrid, 1959 (229 et 211) ; Iñigo Lopez de Mendoza (Marqués de Santillana) Refranes que dizen las viejas tras el fuego, Bizarri Hugo (ed.), Kassel : Reichenberger, 1995 : (58) ; Gonzalo Correas, Vocabulario de refranes y frases proverbiales (1627), éd. de L. Combet révisée par R. Jammes et M. Mir-Andreu, Nueva Biblioteca de Erudición y Crítica, Madrid: Castalia, 2000: A 2173. Abraham Danon («Proverbes judéo-espagnols de Turquie » in Zeitschrift für Romanische Philologie, XXVII, 1903, pp. 72 à 96) fait pour sa part le lien entre ce proverbe et Talmud $B Q 92$ a 'Le chou est arraché (frappé) avec les ronces' commenté par 'les bons paient avec le méchants'.
} 
priori très différent ? Ce sont les textes écrits dans lesquels ces énoncés figurent qui vont nous permettre de le comprendre.

Lors de l'édition critique du proverbier de Mme Flore, j'ai recherché chacune des unités qu'elle avait réunies, tout d'abord dans les listes judéo-espagnoles préexistantes, puis dans certains proverbiers contemporains en turc et en bulgare; puis dans les grands proverbiers espagnols contemporains; dans les proverbiers espagnols et catalans du Moyen Age, du XVI et du XVII ${ }^{\mathrm{e}}$ siècles ; enfin dans les proverbiers arabo-andalous du Moyen Age, généreusement édités dans la deuxième moitié du $\mathrm{XX}^{\mathrm{e}}$ siècle par la critique arabisante en Espagne et plus largement dans les livres de proverbes, maximes et sentences compilés par les auteurs classiques arabes accessibles en traduction.

Dans le domaine du judaïsme, j'ai scruté les parties les plus directement sentencieuses de la Bible (Proverbes, Ecclésiate et Siracide) et quelques traités du Talmud lorsque Mme Flore ou la critique s'y rapportaient, les Pirqe Avot et les alphabets de Ben Sira (dont j'ai parlé ci-dessus) et quelques traités d'exégèse rabbinique. J'ai également consulté les recueils de sentence comme le Miv'har ha-Peninim attribué à Ibn Gabirol.

S'agissant d'Alexandre j'ai consulté à nouveau les textes présentés plus haut. Ce sont ces résultats que j'ai formalisés sur les tableaux 3 et 4.

\section{2 Les textes juifs}

\subsubsection{Les sentences centrales $\mathbf{2}$ et $\mathbf{3}$ et les proverbes cités dans leurs gloses}

En regardant le tableau 3, tout d'abord on percevra du premier coup d'œil la ressemblance entre les réseaux de liens que Mme Flore établit entre les unités et ceux que les textes religieux juifs, Bible et éxégèse rabbinique (Talmud et traités de la Mishna, puis les commentaires rabbiniques judéo-espagnols) établissent entre les mêmes unités.

Comme chez Mme Flore les sentences $\mathbf{2}$ et $\mathbf{3}$ y sont réunies et sont liées aux mêmes exempla.

L'exemplum cité par Mme Flore en commentaire à (E47), fait partie de la légende d'Alexandre telle qu'elle figure dans le Talmud (traité Tamid 32) : Alexandre et la porte du Paradis, épisode qui renvoie explicitement à $\operatorname{Pr}$. 27, 20 « Le sheol et le lieu de destruction ne se rassasient pas et les yeux de l'homme ne se rassasient pas »;

La sentence (K52) «Qui est le riche ? Celui qui se contente de sa part», fait aussi partie de la légende d'Alexandre, insérée dans le même traité talmudique, et portant sur la visite d'Alexandre aux sages du Sud. Israel Levi ${ }^{42}$, qui a comparé les différents textes sur Alexandre, a fait remarquer que dans cet épisode les questions posées aux Brahmanes ou aux Gymnosophistes (les sages du Sud dans le Talmud) étaient en partie différentes de celles figurant dans Plutarque et le Pseudo-Callisthène. Trois questions (Qui est le sage ?, Qui est le puissant ?, Qui est le riche ?) sont prises au traité sapientiel Pirqe Avot (IV, 1). Ces proverbes sont souvent mis en rapport dans les commentaires qui les accompagnent avec d'autres passages des Pirqe Avot (IV, 21 et II, 11) sur la jalousie et la concupiscence qui détruisent l'homme. Ils s'appuient sur Pr. 14, 30 « Le désir pourriture des os ».

Le Me'am Lo'ez $(G n .1,1-6,8)$ reprend ces deux passages à la suite ${ }^{43}$ et ajoute aux sentences $\mathbf{2}$ et 3, dans ses commentaires, kuanti mas tyene mas kere 'plus il a plus il désire' (cf. Flore K27), i el selo lo kita al ombre del mundo (cf. Flore E76), I lo mezmo kada uno buchka dos tantos mas de lo ke tyene $i$ no lo alkansa 'et de la même façon chacun cherche [à

\footnotetext{
${ }^{42} C f$. articles cités supra note 24 .

${ }^{43}$ Cf. D. Gonzalo Maeso et P. Pascual Recuero, Me'am Lo'ez... op. cit., Tome I, $1^{\mathrm{e}}$ partie, dans le chapitre 1 , qui commente le premier verset de Genèse, l'histoire d'Alexandre et ses commentaires occupent les pages 53 à 59 .
} 
obtenir] le double de ce qu'il a et ne peut l'atteindre' (cf. Flore N17), i no dezea mas de lo ke tyene 'et qui ne désire pas plus que ce qu'il a'.

Le lien entre l'oeil de l'homme, la vaine cupidité et la mort se retrouvent également dans $Q o$ 5, 9 « qui aime l'argent ne se rassasie pas d'argent» et Si 14, 9 « L'oeil du cupide n'est pas satisfait de sa part, une avidité mauvaise dessèche son âme ».

On notera que le chapitre X du Miv'har ha-Peninim ${ }^{44}$, lie des énoncés sur l'homme le plus riche qui se satisfait de son sort (sentences 157,158 ) et les injonctions à mépriser les biens de ce monde, à ne pas convoiter ce que l'on n'a pas obtenu (sentence 160,165), à ne pas regarder ce qu'ont les autres (sentence 159), à ce contenter de l'essentiel $(161,171)$ et de ce que le Créateur concède $(159,162)$.

\subsubsection{Les sentences $\mathbf{2}$ et $\mathbf{3}$ et les trois proverbes périphériques}

L'exemplum sur la vanité du désir humain qui ne prend fin qu'avec la mort, représentée métaphoriquement par la terre appelle de façon presqu'inévitable les versets de Gn 3, 19 et $Q o$ 3, 20 « Tu es poussière et tu redeviendras poussière », la Bible fondant la métaphore, dont le proverbe (T31) est une expression judéo-espagnole todo vyene de la tyera $i$ se va a la tyera 'tout vient de la terre et retourne à la terre'.

Le passage de la métaphore de «l'œil vide» au «gosier vide » peut se justifier par la ressemblance du proverbe (K43) 'qui a l'oeil vide n'a jamais le gosier rempli' avec $\operatorname{Pr}$. 13, 25, «Le juste mange et il est satisfait mais le ventre des méchants n'est jamais rassasié » et le Talmud Yoma 74b. Cependant nous verrons infra que d'autres textes ont pu influencer ce changement de métaphore.

Quant à l'extension de ce corpus vers Voir et Désirer (V4), il trouve son fondement dans le texte biblique même où « tu ne lèveras pas les yeux sur la femme de ton voisin... » se traduit par 'tu ne convoiteras pas...' (Ex. 20, 17 et $L v .5,21)$. Dans le Midrash des $X$ commandements et le Livre Précieux de la Salvation ${ }^{45}$, comme dans le Me 'am Lo'ez, les exempla sur l'homme tenté par une femme interdite qui détourne le regard, ferme les yeux ou même se les crève sont légion. L'exemple par excellence est Joseph devant la femme de Putiphar. Le lien direct entre la vue et le désir est également inscrit dans Qo 6, 9 : «Meilleur est ce que les yeux voient que ce que l'âme désire ». La prégnance de ces textes et la force de la métaphore, expliquent sans doute l'attraction subie par le proverbe (K32) 'Qui regarde les gens ne vit jamais content', qui de 'bien faire et laisser dire', leçon que lui donne aussi Mme Flore (il ne faut pas se soucier de l'opinion des gens) a acquis le sens de 'il ne faut pas convoiter le bien d'autrui sous peine de causer son propre malheur', sens qu'il n'a pas acquis en Espagne, par exemple.

\section{2. 3 Les sentences $\mathbf{1}$ et $\mathbf{6}$ et les proverbes et bénédictions qui leur sont liées}

Les textes bibliques et religieux en hébreu en rapport avec les énoncés de la partie de gauche étaient, je l'ai dit, directement évoqués par Mme Flore : les désignations de Dieu, les bénédictions qui lui sont adressées, la première phrase du Kaddish (citée en hébreu), la bénédiction redoutable qui accueille l'annonce d'un décès barukh dayan ha-emet, 'béni soit

\footnotetext{
${ }^{44} C f$. édition de D. Gonzalo Maeso, op. cit., pp. 66 - 67 sentences 155 à 172.

${ }^{45}$ Cf. Amparo Alba Cecilia (ed.), Midras... op. cit. Le commentaire au septième commandement « Tu ne commettras pas l'adultère » $(E x, 20,14)$ cite l'exemple de R. Mattia b. Jeres (Exemplum n ${ }^{\circ} 136$ tiré du Talmud de Jérusalem, Sab 3) qui nunca en su vida había levantado los ojos hacia la mujer de su prójimo, 'n'avait jamais de sa vie levé les yeux sur la femme de son prochain'. Tenté par le diable, il se crève les yeux, et lorsque l'archange Raphaël vient le soigner, il refuse de recouvrer la vue afin de ne plus pouvoir être tenté.
} 
celui qui rend des sentences de vérité ${ }^{46}$, l'exemple de Job enfin qui introduit l'idée de l'apparente injustice de la rétribution. Dans le monde judéo-espagnol la mort et la malchance sont interprétées comme une punition des péchés. Le lien est fait par Mme Flore puisqu'elle cite comme ayant un rapport direct un proverbe hébreu sur l'injustice en ce monde (commentaire à E25) El bueno tyene lo negro i el negro tyene lo bueno ${ }^{47}$, 'le bon obtient ce qui est mauvais et le mauvais ce qui est bon'. Elle y ajoute une glose explicite qui a valeur de sentence : El byen i el mal en el mundo no es syempre espartido sigun los meritos $i$ las kulpas de kada uno, 'Le bien et le mal dans ce monde ne sont pas toujours répartis selon les mérites et les fautes de chacun'. Or ce dernier énoncé figure tel quel dans un commentaire du Talmud (Berakhot 7a) à propos des trois questions de Moïse sur la rétribution, qui, s'appuyant sur différents passages bibliques (Ex. 34, 7 ;Dt. 24, 16 ;Ex. 33, 19) concluent à 1'impossibilité, même pour Moïse, de comprendre les motivations divines.

De nombreux exempla des traités rabbiniques et du Talmud sont consacrés à ce chapitre. Amparo Alba et Angeles Navarro ${ }^{48}$ rapportent l'exemplum de 1'homme pieux enterré humblement tandis que la foule se presse à l'enterrement du fils du collecteur d'impôts qui figure dans les recueils cités plus haut et dans le Talmud (Hagiga 2, 2). Elles y ajoutent l'exemplum du saint paralytique qui avait reçu le don de pouvoir se lever lorsqu'on enterrait un juste, qui porte aussi sur l'apparente injustice de la rétribution, et qui figure dans le Sefer Shaashuim, 'Livre des divertissements' de Joseph ben Meir Ibn Zabarra (auteur juif d'Espagne, au XIII ${ }^{\mathrm{e}}$ s).

\section{3 Les textes anciens}

Si nous regardons ce que les textes anciens non-juifs circulant à l'époque médiévale nous apprennent, nous trouvons la présence de l'épisode d'Alexandre et la porte du Paradis seulement dans les recensions $g$ et $e$ du Pseudo-Callisthène. Elles proviennent d'après la critique $^{49}$, de la version syriaque, pour laquelle Israel Levi suppose une version araméenne antérieure. Cette recension donne naissance à la plupart des versions arabes du PseudoCallisthène. L'opuscule latin Alexandri magni iter ad Paradisum qui contient l'exemplum de l'oeil, est considéré comme d'origine juive ${ }^{50}$. Quant à la visite chez les Brahmanes (ou Gymnosophistes ou Sages du sud) elle ne comporte pas, dans le Pseudo-Callisthène, ni dans de Proeliis, ni dans les Res Gestae de Julius Valère, ni chez Plutarque ${ }^{51}$, la question 'Qui est le riche ?' (K52), tirée des Pirqe Avot. Cependant on trouve cette dernière comme sentence indépendante chez Publilius Syrus ${ }^{52}$. On trouve aussi, chez Diogène Laërce (VI - Antisthènes

\footnotetext{
${ }^{46}$ Cette bénédiction en hébreu est intégrée au judéo-espagnol et très utilisée, $c f$. à ce sujet David M. Bunis, $A$ lexicon of the Hebrew and Aramaic Elements in Modern Judezmo, Jérusalem : Magnes Press, 1993, n 699.

${ }^{47}$ Traduction qu'elle donne elle-même à la formulation hébraïque qu'elle cite également ha-tov we-ra' lo, hara'we-tov lo.

${ }^{48}$ Voir leur article cité plus haut (note 6) «Del cuento rabínico... ». Elles renvoient pour les exempla cités aux chapitre IX du Midrash des Dix Commandements (op. cit., pp. 95 - 96) et I, 14 du Livre Précieux de la Salvation (op. cit., pp 119-121).

${ }^{49}$ Voir notamment George Cary, op. cit.; Israel J. Kazis (ed.), op. cit. ; Moses Gaster, Studies and Texts... (op. cit.), vol. 2, p. 814 ; et les articles d'Israël Levi, cités supra, note 24.

${ }^{50}$ I. Levi, R.E.J., II, 1881 (op. cit. note 24).

${ }^{51}$ idem et Jean - Pierre Callu, « Alexandre dans la littérature latine de l'Antiquité tardive » in Laurence Harf Lancner \& alii (op. cit.), pp. 33 à 50 ; idem Laurence Harf Lancner, « Alexandre et l'Occident Médiéval », pp. 15 à 19. Plutarque, op. cit. ; éditions et traductions du Pseudo-Callisthène, par G. Bounoure et B. Serret (op. cit.) et par A. Tallet Bonvalot (op. cit).

${ }^{52}$ Cf. Publilius Syrus, « Sentencias », Victor J. Herrero LLorente (éd.), Suplementos de Estudios Clásicos, $\mathrm{n}^{\circ} 7$, Madrid, 1963, p. 296, n 626 : Quis plurinum habet? Is qui omnium minimum cupit. Elle n'est pas reliée à Alexandre.
} 
5), la métaphore du fer usé par la rouille comme l'homme par l'envie ( $c f . \operatorname{Pr}$. 14, 30 : le désir, pourriture - ou carie- des os). Il s'agit là d'énoncés isolés, sans rapport direct avec Alexandre.

Le texte du Pseudo-Callisthène ${ }^{53}$ donne un énoncé complet de la réponse d'Alexandre à Dandamis (Livre III, 13), proche des sentences 1 et $\mathbf{6}$ de Mme Flore : «Pas de vagues sur la mer si le vent n'a pas soufflé, pas d'agitation dans les arbres si l'air ne les a pas secoués, pas d'activité chez les hommes sinon sous l'effet de la Providence céleste ${ }^{54}$. A la cupidité insatiable d'Alexandre, que lui reprochent les sages et à l'inéluctabilité de la mort qui en montre la vanité, Alexandre oppose la volonté divine à laquelle il obéit. Cette distribution recoupe en partie celle de Mme Flore, mais ici le lien est direct.

Dans les corpus arabes anciens deux hadith du Prophète se rapportent respectivement à l'énoncé 3 (E47) et à (V4) 'Voir et désirer'.

Fernando de la Granja ${ }^{55}$ a montré dans une étude sur l'origine arabe de l'expression llenar el ojo, 'remplir l'œil', que le proverbe utilisé par al-Maydani «Peut-être trouves-tu licite de te remplir l'oeil de ce qui appartient à un autre » est lié à un hadith. Reposant sur un énoncé en rapport avec une anecdote de la vie du Prophète : «Dieu n'a pas créé un seul de ces hommes qu'il n'ait rempli leurs deux yeux de la poussière de cette poignée de terre » qui évoque les impies, ce hadith s'est transmis sous différentes formes : "Seule la poussière est capable de remplir (fermer) le ventre (l'oeil, la bouche) de l'être humain ». Fernando de la Granja rapproche opportunément ce texte au français «avoir les yeux plus gros que le ventre » et à l'espagnol Se te ha llenado el papo antes que el ojo, 'ton gosier s'est rempli avant ton oeil ${ }^{56}$. On y voit le passage de l'œil qui ne se remplit pas au ventre qui ne se remplit pas et on retrouve «le gosier» du proverbe judéo-espagnol (K43) relié à «l'oeil vide» de (E47). Le fait qu'en espagnol moderne et en français les seules formes liées à cet énoncé qui se soient maintenues ne fassent aucune allusion à la terre du tombeau qui seule remplit l'oeil avide, a sans doute à voir à la fois avec le texte arabe passé en espagnol et avec $P r$. 13, 25 ( $c f$. supra) sur lequel peut se fonder sa leçon (en l'absence de l'exemplum d'Alexandre).

Contrairement aux textes juifs, les textes anciens n'expliquent pas les relations intertextuelles bâties par les renvois de proverbe à proverbe et les gloses de Mme Flore. Ils expliquent par contre la présence de ces énoncés dans le proverbier espagnol, l'ancienneté de certaines unités figurant dans les textes juifs, et l'évolution en Espagne et en France de la métaphore de «l'oeil ouvert » au « gosier » ou au « ventre ouvert ».

\section{4 Les traditions arabes sur Alexandre}

\section{4. 1 La version aljamiado-morisca du Pseudo-Callisthène}

En dehors du lien fait par les écrits religieux juifs, et celui, partiel, que crée la visite aux sages de l'Inde et à leur chef Dindimus dans le Pseudo-Callisthène, un lien subsiste-t-il qui unit d'autres énoncés d'Alexandre et lequel ? Les renseignements trouvés ont été synthétisés dans le tableau 4.

Les légendes aljamiado-moriscas et les versions arabes du Pseudo-Callisthène sont plus complètes et plus proches du réseau de liens tissé par Mme Flore en ce qu'ils reprennent

\footnotetext{
${ }^{53}$ Cf. la recensio vetusta traduite par A. Tallet Bonvalot (op. cit.), et la traduction de la version grecque protobyzantine de G. Bounoure et B. Serret (op. cit.) Livre III, 13, dont le texte est repris ici.

${ }^{54}$ Le Sefer ben Gurion, qui reprend des passages du Pseudo-Callisthène, le formule ainsi : Y la mar y los arvoles no se menean sin viento, y ansi el ombre no se aqueda y non repoza sin licencia de el Alto, 'et la mer et les arbres ne bougent pas sans vent et l'homme ne reste pas immobile et ne se repose pas sans la permission du TrèsHaut' ( $c f$. M. Lazar, Sefer ben Gurion... op. cit., p. 104) ; cf. également infra le texte aljamiado-morisco.

${ }^{55}$ Fernando de la Granja, «Llenar el ojo », Al-Andalus, 41 (2 ), 1976, pp. 445-459.

56 idem, note 44.
} 
non seulement l'épisode des sages, mais aussi l'épisode de la source du Paradis et l'exemplum de l'objet qui n'a pour contrepoids que la terre du tombeau.

Un texte d'une légende aljamiado-morisca sur Alexandre (en fait une version du Pseudo-Callisthène) éditée par Guillén Robles à partir d'un manuscrit du XIV ${ }^{\mathrm{e}}$ siècle conservé à Madrid Leyenda aljamiado morisca sobre Alejandro el grande, présente à ce sujet un grand intérêt.

Le texte contient l'épisode d'Alexandre (Dulkarnain) et de la pierre, métaphore de l'ambition, qui lui est remise près de la fontaine d'éternité et dont l'énigme est élucidée par Al-Khadir, compagnon d'Alexandre (comme dans la plupart des versions arabes): après avoir rappelé la quête toujours insatisfaisante de nouveaux territoires $Y$ no te fartarias de nada 'Tu ne te rassasierais de rien', il fait le lien avec la pierre Asi como no se farta aquesta piedra (...) asi nunca te fartarás i no se fartará tu ojo de ninguna cosa, 'De la même façon que cette pierre ne se rassasie pas (...) tu ne te rassasieras jamais et ton oeil ne se rassasiera de rien'. Ici le lien est fait entre l'objet donné et l'oeil qui ne se rassasie pas. Cet énoncé est immédiatement précédé de l'avertissement $Y$ todo lo que has ganado y has enseñoriado (...) y has allegado lo dexarás 'Et tout ce que tu as gagné, dominé (...), obtenu, tu le laisseras'. Et il est immédiatement suivi de l'annonce de la mort : $Y$ tu tornamiento será a aquesta tierra (...) y no será tu parte del mundo (después de muerto) mas de aqueste palmo de tierra aquel sobre que duermes en el dia de hoy y no podrás por menos de tornar a él y dormir de debaxo del, 'Et tu retourneras à cette terre ; et ta part du monde (après ta mort) ne sera plus que cet empan de terre sur lequel tu dors aujourd'hui et tu ne pourras éviter de retourner à lui et de dormir dessous' ${ }^{57}$. Comme dans le proverbier de Mme Flore, l'exemplum de la version arabe du Pseudo-Callisthène enchaîne les sentences sur le retour à la terre du tombeau, l'envie insatiable, la vanité de ces conquêtes et du désir de possession qui échoue devant la mort et utilise la métaphore de l'oeil qui ne se rassasie pas.

Mais ce qui est le plus remarquable, c'est que dans ce passage, avant d'élucider la métaphore de la pierre et de l'oeil, Al-Khadir loue longuement la toute puissance divine et ses merveilles employant une formulation très proche de (P11) (cf. supra: Para maraviyas del Dyo no ay kavo, 'pour les merveilles de Dieu il n'y a pas de fin') qui semblait incongru dans le contexte judéo-espagnol. La formulation du texte aljamiado morisco est en effet: Allah da maravillas a sus criaturas y sus cosas formadas son todas maravillas "Allah donne des merveilles à ses créatures et les choses qu'Il a formées sont toutes des merveilles'.

On trouve également dans ce texte aljamiado-morisco les questions aux Gymnosophistes (ou Brahmanes) d'Alexandre mais la question «Qui est le riche ?» n'y figure pas. Un deuxième ensemble est formé dans ce texte de source arabe par le récit de la visite aux Sages et à Dandamis / Dindimus, relatée par Alexandre dans sa lettre à Aristote sur les merveilles de l'Inde, qui correspond au chapitre III du Pseudo-Callisthène. On y retrouve la condamnation par les sages de la poursuite incessante des biens de ce monde et de la recherche du pouvoir. Les conseils sont proches dans leurs formulations des énoncés de Mme Flore : bástete con lo que vives día empués día; y te hartará lo poco, si te conformas con ello ; y no desee tu presona allegar fortuna, 'contente-toi de ce qui te permet de vivre jour après jour; tu te rassasieras de peu si tu acceptes de t'en contenter; et que ta personne ne cherche pas à obtenir la fortune'. Ils conseillent de renoncer au monde et à ses richesses, de se contenter de ce qui est donné par Dieu et de l'en remercier : hemos aborrecido el mundo y su cobdiçia, 'nous avons renoncé au monde et à sa cupidité'; agradece a Allah lo que te da, 'rends grâces à Dieu pour ce qu'il te donne' ; nos contentamos con lo que tenemos de esto, 'nous nous contentons de la part que nous avons de cela'; nuestros padres agradecian lo que les era dado, nos pères étaient reconnaissants pour ce qui leur était donné'.

\footnotetext{
${ }^{57}$ G. Robles, Leyendas... p. 175, 177 et $178-179$.
} 
L'apologie de l'action humaine que fait Alexandre en réponse, est ici entièrement régie par la toute puissance divine. Alexandre obéit à Dieu et sa justification repose exactement sur la même formulation et les mêmes exemples que dans les textes grecs.

Les sages utilisent également dans les conseils qu'ils donnent à Alexandre l'expression métaphorique Llena tu ojo, 'remplis ton oeil' 58 .

Le texte reproduit dans le tableau 3 montre comment sont reliés les énoncés de la partie droite et ceux de la partie gauche supérieures, tous appuyés sur un petit nombre d'épisodes très concentrés ${ }^{59}$. La légende de source arabe ajoute aux liens faits par le PseudoCallisthène grec la métaphore de l'oeil qui ne se rassasie pas, le retour de l'homme à la terre du tombeau, et surtout la célébration enthousiaste d'une toute-puissance divine qui comble les hommes de ses merveilles, de sa justice et de sa bienveillance. L'idée de la rémunération des justes et des méchants en est absente, ainsi que l'impénétrabilité de la volonté divine et la crainte que sa toute-puissance engendre.

\section{4. 2 Les autres textes médiévaux de source arabe sur Alexandre}

Tout ce qui précède ne nous dit pas pourquoi, dans le proverbier de Mme Flore, les deux énoncés de gauche $(\mathbf{1} \& \mathbf{6})$ et les deux de droite $(3 \& 2)$ se trouvent fortement reliés, alors que les deux derniers proverbes d'Alexandre $(\mathbf{4} \& \mathbf{5})$ ne sont pas liés entre eux ni aux autres.

On voit également sur le tableau 3 (partie inférieure) que les deux proverbes écartés se trouvent liés, quant à eux, dans et par un autre corpus sur Alexandre. Celui qui figure dans Libro de los buenos proverbios issu, comme nous l'avons dit supra du livre arabe des dits et enseignements des philosophes (Kitab adab al falasifa), traduit en hébreu à la fin du XII ${ }^{\mathrm{e}}$ siècle ou au début du XIII ${ }^{\mathrm{e}}$ par al-Harizi sous le nom de Mussre ha-Filosofim. Ces éléments se retrouvent en partie, rappelons-le, dans d'autres textes médiévaux comme Bocados de Oro et la Disciplina Clericalis. Les deux énoncés, 4 (S29) se arastó lo seko kon lo vedre, 'le vert a été arraché avec le sec' et 5 (L50) Las dadas son tomadas 'les [choses] données sont [re]prises', figurent dans la lettre qu'Alexandre écrit à Olympias pour la consoler de sa mort prochaine et dans la réponse de celle-ci. La formulation est répétée à peu près à l'identique à deux reprises et lie à chaque fois les deux énoncés dans le texte médiéval : Non vedes que los árboles verdes y fermosos que fazen muchas fojas i espessas i yevan mucho fruyto y a poco de tiempo quebrántansse sus ramas y cáense sus fojas y su fructo [...]. Madre, non vedes las yerbas floridas que amanesçen verdes y anochesçen secas [...] Madre, vistes quien diesse $i$ non toliesse..., 'Ne vois-tu pas que les arbres verts et beaux qui forment beaucoup de feuilles et épaisses et portent beaucoup de fruit et [dont] en peu de temps se cassent les branches et tombent les feuilles et le fruit [...]. Mère, ne vois-tu pas les herbes fleuries qui se lèvent vertes le matin et se couchent sèches le soir [...]. Mère, as-tu déjà vu que l'on donnât sans reprendre...; Fue verdat dixietes que los rramos verdes y fermosos a secarse an, $i$ las fojas a cers'an i el que dió, tomó, ca todo es vanidat, 'Tu as dit vrai, que les branches vertes et belles doivent sécher et que les feuilles doivent tomber et que celui qui a donné, a repris, car tout est vanité'.

\footnotetext{
58 idem, p. 233.

${ }^{59}$ Les énoncés comme on l'a vu dans les notes précédentes se concentrent autour de la source du Paradis (pages 177 à 179) et de la visite aux Sages (pages 221 à 226). Trois références sont excentrées « remplis ton oeil» et « rends grâce à Allah de ce qu'il te donne » (p. 233 ) et «Nos pères rendaient grâce pour ce qui leur était donné » qui fait partie d'une première visite d'Alexandre aux Sages (p. 157). « et ton oeil ne sera rassasié par rien » qui fait partie de l'épisode de la pierre donnée à la source du Paradis est répété dans l'épisode des Sages (p. 227). Pour rendre les résultats plus clairs les énoncés du tableau 4 ont été distribués en suivant la répartition des énoncés chez Mme Flore ( $c f$. Tableau 1).
} 
La métaphore du vert et du sec illustre ici la réversibilité du destin, menant toutes choses implacablement à la mort, quelle qu'en soit la beauté, la jeunesse ou la puissance. Alexandre mourant avant le temps illustre la vanité de ce monde. Chez Mme Flore (4) la métaphore signifie également la destruction absolue et inéluctable, peut-être en relation avec Is 40, 7 «La fleur fane et l'herbe sèche quand le souffle de Dieu la frappe (certainement, l'herbe est le peuple) ». Cependant, elle ne lui permet pas de relier cet énoncé à la toute puissance divine ( $\mathbf{6}$ et $\mathbf{1}$ ), ni aux énoncés sur la rétribution apparemment injuste ${ }^{60}$.

«Ce qui est donné et repris », représente dans Libro de los buenos proverbios, la toute-puissance du créateur qui donne la vie et qui la reprend, comme dans $J b 1,21$ « Le Seigneur a donné, le Seigneur a repris ». Malgré la proximité du texte biblique, l'énoncé 5 n'a pas de rapport avec l'énoncé 4, pour Mme Flore, ni avec les autres énoncés proverbiaux sur Alexandre et ceux qui leur sont liés. L'énoncé reçoit une leçon tout à fait différente, «On récolte ce que l'on a semé ». L'opposition entre dar 'donner' / tomar 'recevoir', 'prendre', 'reprendre', amène ici une modification du sens « ce qui est donné est rendu », 'on est payé de sa pièce', 'on reçoit en retour le bien ou le mal que l'on a fait'. Le sens figuré et la leçon du proverbe se sont considérablement modifiés.

Visiblement le texte de buenos proverbios n'a pas joué le même rôle que la version arabe du Pseudo-Callisthène, ni surtout que l'éxégèse talmudique. Il n'a pas eu la même postérité dans la structuration de l'expérience sapientielle judéo-espagnole. Peut-être parce que la diffusion du texte s'est trouvée interrompue dans la diaspora : le texte hébreu de alHarizi ne semble pas, en effet, avoir fait l'objet d'éditions dans l'ex-Empire ottoman. Peutêtre aussi parce que, contrairement aux autres textes sur Alexandre, buenos proverbios ne s'appuie pas sur les textes talmudiques, n'a pas bénéficié de commentaires rabbiniques postérieurs, ni de l'existence d'exempla intermédiaires, qui, en assurant son articulation à l'appareil sapientiel juif, aient pu maintenir les liens des énoncés entre eux. Les énoncés du Libro de los buenos proverbios ont survécu dans le proverbier judéo-espagnol mais, privés de l'appui textuel qui organisait leur réseau signifiant, ils ont perdu les liens qui les unissaient au Moyen Age. Ils ont rejoint d'autres réseaux interprétatifs et (du moins dans le cas de 6) ont tout à fait changé de sens.

\section{Conclusion}

Ainsi le proverbier glosé de Mme Flore, qui apparaît a priori comme une suite aléatoire d'énoncés est en réalité articulé et fortement structuré, de manière sous-jacente, sans que la locutrice n'en ait elle-même conscience, par des ensembles textuels anciens. Par le jeu des correspondances entre les énoncés, leur sens propre et leur sens figuré, leurs contextes d'emplois, les leçons qu'ils contiennent et les rapports qu'ils entretiennent avec les corpus d'exempla, ces textes organisent l'ensemble transmis. Dans le cas d'Alexandre la structuration principale des énoncés repose sur la Bible, le Talmud et l'éxégèse rabbinique en un réseau serré de correspondances et de renvois qui font de cet ensemble d'énoncés un tout cohérent en accord avec les fondements même du judaïsme. Ils s'appuient sur un écrit dont les différents strates assurent la stabilité, du plus fixe et ancien, le texte biblique, au plus instable et récent, le proverbier. L'exégèse talmudique, les recueils d'exempla rabbiniques, les commentaires rabbiniques judéo-espagnols en sont des strates intermédiaires. Le proverbier et le contier, comme la faculté de creer proverbes et exempla, sont la face orale de ces textes. Mme Flore n'a pas de formation religieuse autre que superficielle, elle n'a eu d'accès direct ni au Talmud, ni au Me'am Lo'ez, ni aux Pirqe Avot, ni aux recueils d'exempla rabbiniques, pas plus qu'elle n'a lu le Pseudo-Callisthène ou la Leyenda aljamiado morisca, le Libro de los

\footnotetext{
${ }^{60}$ Comme c'est notamment le cas en espagnol (cf. supra § 3.4 et note 41 ).
} 
buenos proverbios, Bocados de oro ou encore Mussre ha Pilosophim. Ces derniers en effet n'étaient pas à la mode et ne faisaient plus partie des textes édités accessibles, et, par conséquent, des lectures de la bourgeoisie judéo-espagnole.

Ce qui demeure chez Mme Flore est ce qui a été transmis par la tradition orale de ces corpus.

Pourtant, son commentaire des proverbes suit parfaitement la logique des commentateurs rabbiniques et parvient à une éxégèse complexe s'appuyant implicitement sur le Talmud.

Il apparait, d'autre part, que les versions juives et arabes du Pseudo-Callisthène et les textes arabes sur Alexandre qui circulaient en Espagne au Moyen-Age ont contribué également à la structuration de cet ensemble et expliquent l'inclusion ou l'exclusion de certains énoncés dans les réseaux interprétatifs de Mme Flore.

Le proverbier de Mme Flore garde donc des traces, implicites, des relectures croisées des textes du Moyen-Age. C'est probablement la variation des commentaires musulmans qui explique le passage de «l'œil» à la «bouche» et au «ventre » ou au «gosier» dans la métaphore sur l'insatiable désir de l'homme, mais cette leçon est aussi soutenue par le texte de $\operatorname{Pr} 13,25$. On notera l'importance des métaphores dans le maintien du sens et dans le maintien de la mémoire en réseaux d'énoncés.

Il n'y a pas ici de coupure entre l'oral et l'écrit, la parole et le texte, les savoirs populaires et les savoirs savants, le profane et le clerc. Le proverbe et l'exemplum appartiennent certes à l'oral le plus formel et le plus proche de l'écrit, mais leur usage relève du quotidien et du domaine de la parole. Le discours proverbial loin de se constituer dans un domaine indépendant de l'écrit, repose sur lui. Inversement, l'oral actualise l'écrit et l'acquisition des proverbes et des exempla en rapport avec les situations quotidiennes permet aux profanes d'accéder aux savoirs savants qui structurent profondément l'identité du groupe et, indirectement, redonnent une pertinence aux textes sous-jacents. Au-delà du commentaire des situations quotidiennes, c'est l'organisation textuelle et interprétative qui structure la mémoire du groupe et qui fournit une grille de lecture du monde, qui est transmise avec le proverbier. L'oral et l'écrit apparaissent ici indissociablement liés.

Marie-Christine Bornes-Varol

PU Judaïsme séfarade et Judéo-espagnol

Dépt. Etudes Hébraïques et Juives

INALCO - Paris \& UMR 8099, Langues, Musiques, Sociétés

\section{BIBLIOGRAPHIE ${ }^{61}$}

\section{Corpus Hébreu ancien et médiéval}

Aggadoth du Talmud de Babylone - La Source de Jacob - 'Ein Yaakov, (1982), A. ElkaïmSartre éd., Paris : Verdier.

Biblia de Jerusalén, (1975), Madrid : Alianza editorial.

\footnotetext{
${ }^{61}$ Il s'agit ici de la bibliographie générale de l'ouvrage et des références des ouvrages cités dans la section $10 \mathrm{du}$ Chapitre III ; elle est à compléter des listes de références contenues dans les notes des proverbes, présentées section 9 du chapitre III.
} 
COHEN, A. (Rabbin), (1986), Le Talmud - Exposé synthétique du talmud et de l'enseignement des Rabbins sur l'éthique, la religion, les coutumes et la jurisprudence, Paris : Payot.

El testamento de moral de Rabenu Aşer, (1964), Traduit par le rabbin Y. Rofe, Istanbul : Güler basımevi. [début $\mathrm{XIV}^{\mathrm{e}} \mathrm{s}$.]

IBN GABIROL Š(e)lomó, (1977) Selección de perlas (Mibhar ha-P(e)nînîm), versión española con introducción y notas por D. Gonzalo Maeso, Biblioteca nueva Sefarad, Barcelona : Ameller.

Pirke Avot-Libro de moral cudia, (1954), Traduction et commentaire de N. Behar, Istanbul : Güler Basımevi.

Pirké Avot, (1993), Traduction et commentaire de C.- A. Gugenheim, in La Michna-Tome $\mathrm{XV}$, Paris : CLKH.

ROMERO Elena, (2001) Andanzas y prodigios de Ben-Sirá - Edición del texto judeoespañol y traducción del texto hebreo, Madrid : CSIC.

SCHWAB, Moïse, (1907), «Version espagnole des Alphabets de Ben-Sira » in Revue des Etudes Juives, Tome LIV, pp.107 à 112.

SCHWARZWALD (RODRIGUE), Ora, (1989), « The ladino Translations of Pirke Aboth », Eda velashon, 13, Jérusalem : The Magnes Press. 


\section{Corpus Arabe}

AL-ĀBŚĪHĪ, Śihāb-ad-dīn Āhmad, (1899 \& 1902), Al-Mostatraf - Recueil de morceaux choisis çà et là dans toutes les branches de connaissances réputées attrayantes, Traduction de G. Rat, II tomes, Paris : Ernest Leroux.

Al-Mostatraf (Mostatref) cf. AL-ĀBŚĪH̄̄, Śihāb-ad-dīn Āḥmad

BEN CHENEB, Mohammed, (2003), Proverbes de l'Algérie et du Maghreb, Paris : Maisonneuve et Larose -INAS.

BLACHERE, Régis, (1966), Histoire de la littérature arabe des origines à la fin du XV siècle de J.-C., Tome III, Paris : Adrien Maisonneuve.

BURCKHARDT, Johann Ludwig, (1830), Arabic Proverbs or the manners and customs of the modern Egyptians, illustrated from their proverbial sayings current at Cairo, W. Ouseley éd., Londres : J. Murray.

FREYTAG, Georg W., (1838 - 1843) Arabum Proverbia, Bonne : A. Marcum. (2 vols).

GARCIA GOMEZ, Emilio, (1970a) « Hacia un refranero arábigo andaluz -I.- Los refranes de Ibn Hišām al-Lajmī», in Al Andalus, XXXV, pp. 1 à 68.

- (1970b) « Hacia un 'refranero' arábigo andaluz -II.- El refranero de Ibn 'Āsim, en el ms. londinense», in Al Andalus, XXXV, pp. 241 à 314.

- (1971), «Hacia un 'refranero' arábigo andaluz -III.- Los refranes poéticos de Ben Šaraf (texto inédito) in Al-Andalus, XXXVI, pp. 255-328.

- (1972a), «Hacia un 'refranero' arábigoandaluz - IV. - Los proverbios rimados de Ben Luyūn de Almería (1282-1349)», in Al Andalus, XXXVII, pp. 1 à 75.

- (1972b), « Hacia un 'refranero' arábigoandaluz - V. - Versión del libro sobre refranes de al'Iqd al-farìd (s. X). Preliminares y refranero de Aktam y Burzuyumihir», in Al Andalus, XXXVII, pp. 249 à 323.

GUTAS, Dimitri, (1975), Greek Wisdom Literature in Arabic Translation - A Study of the Graeco-Arabic Gnomologia, American Oriental Series, vol. 60, New Haven (Connecticut): American Oriental Society.

MARUGAN GÜEMEZ, Marina, (1994) El refranero andalusí de Ibn 'Asim al-Garnati, Hiperión, Madrid, 1994.

THA'ĀLIBĪ, (1987), La beauté est le gibier des cours, Al-I'jâz wa'l-ijâz, Anthologie de paroles et de maximes, traduite de l'arabe et présentée par O. Petit, Paris : Sindbad.

\section{Corpus Textes autres}

DIOGENE LAËRCE (1999), Vies et doctrines des philosophes illustres, trad. française sous la direction de M.-O. Goulet-Cazé, J.-F. Balaudé et alii éds., coll. Classiques Modernes, s.l. : La Pochothèque. $\left[1^{\text {ère }}\right.$ moitié du $\mathrm{III}^{\mathrm{e}}$ s. après JC]

EYÜBOĞLU E. Kemal, (1973), On üçüncü yüzyıldan günümüze kadar Şiirde ve Halk Dilinde Atasözleri ve Deyimler, Istanbul : Doğan Kardeş Maatbacılık (2 vol.). 
MALOUX, Maurice, (1960), Dictionnaire des proverbes sentences et maximes, Paris: Larousse.

MORAWSKI, Joseph (1924), Les diz et proverbes des sages, Bibliothèque de la Faculté des Lettres, $\mathrm{II}^{\circ}$ série, Paris : Les Presses Universitaires de France.

- (1925), Proverbes français antérieurs au $X V^{\circ}$ siècle, Paris : E. Champion.

NAU, François, (1986) Histoire et Sagesse d'Ahikar l'Assyrien, Paris : Berg international

SELMER, C., (1940), “An unpublished latin collection of Pseudo-Aristotelian Paroimai”, Speculum, 15, pp. 92-4.

\section{Corpus séfarade}

ALBARRAL ALBARRAL, Purificación, (2001), Una cala en la literatura religiosa sefardí : La Almenara de la luz, Grenade : Université de Grenade.

ALKALAY, Arye, (1984), Dichos i refranes sefaradies, D. Benveniste éd., Jérusalem : Ed. de la Comunidad Sefardita de Jerusalén.

AŞER (Rabenu) cf. El testamento de moral...

BEHAR Nisim, (1954), Pirke Avot - Livro de moral cudia, Istanbul : Güler Basımevi.

- (1967), El Gid para el pratikante, Istanbul : Güler Basımevi.

BENAZERAF Raphaël, (1978), Recueil de refranes judéo-espagnols du Maroc, Paris : chez l'auteur.

CANTERA ORTIZ DE URBINA, Jesús (2004), Diccionario Akal del refranero sefardí, Madrid : Akal.

CARRACEDO, Leonor \& ROMERO, Elena, (1981), «Refranes publicados por Ya'acob A. Yoná (edición concordada) y Bibliografía del Refranero sefardí » in Sefarad - (Estudios Sefardies 4), XLI, fasc. 3.

DANON, Abraham, (1903), «Proverbes judéo-espagnols de Turquie » in Zeitschrift für Romanische Philologie, XXVII, pp. 72 à 96.

FOULCHE - DELBOSC, Raymond, (1895) «Proverbios judeo-españoles recogidos en Constantinopla, Adrianópolis y Salónica », in Revue Hispanique, $2^{\mathrm{e}}$ année, $\mathrm{n}^{\circ} 4$, Paris, pp. 312 à 352.

GUTWIRTH, Eleazar, (1992) «A Medieval Manuscript of Gnomic Verse in Judeo-Spanish aljamía » in Circa 1492 - Proceedings of the Jerusalem Colloquium : Litterae Judaeorum in Terra Hispanica, I. Benabu éd., Jérusalem : The Hebrew University \& Misgav Yerushalayim, pp. 98-108.

KAYSERLING M., (1889), Refranes o proverbios españoles delos judios españoles, Budapest : Posner \& Fils. 
- (1890) «Proverbios judeo-espanoles recogidos en Budapest, Belgrado y Roustchouk », Biblioteca española portuguesa judaica, Strasbourg.

- (1897) «Quelques proverbes judéo-espagnols », in Revue Hispanique, IV, Paris / N.Y.

KOLONOMOS, Djamila, (1978) Poslovice, Izreke i Priče sefardskih jevreja makedonije Proverbs, Sayings and Tales of the Sephardi Jews of Macedonia, Belgrade : Federation of the Jewish Communities in Yugoslavia.

LIDA, Denah, (1958), «Refranes de los judeo-españoles de Esmirna » in Nueva Revista de Filología Hispánica, Tome XII, pp. 1 à 35.

LEVY Isaac Jack, (1969), Prolegomena to the Study of the Refranero Sefardi, New York: Las Americas Publishing Co.

LEON, Luis, (2001) Refranes y expresiones sefaradies - De la tradición judeo-española de Esmirna, Buenos Aires : Milá.

MOSCUNA, A., (1897) «Spaniolische Sprichtwörter aus Tatar-Bazardzyk in Ost-Rumelien », in Der Urquell, (2 parties : I, pp. 84 à 87 ; II, pp. 204 à 205).

PERAHYA Klara, DE TOLEDO Suzi, DANON Suzi, ENDER Fani, (1994), Erensya Sefaradi, Istanbul : Gözlem.

Poslovice i Izreke - Proverbs and Sayings of the Sephardi Jews of Bosnia and Herzegovina, (1976), Dj. Kolonomos et alii (éd.), Belgrade : Federation of Jewish Communities in Yugoslavia, Belgrade.

SAPORTA Y BEJA, Enrique, (1978), Refranes de los judíos sefardies, Biblioteca Nueva Sefarad, Vol. V, Barcelona : Ameller.

SUBIRA, José (1954), "Romances y refranes sefardíes" in Estudios dedicados a Menéndez Pidal, tome V, Madrid, pp. 319 à 333.

\section{Littérature judéo-espagnole contemporaine}

ALONSO GARCIA, Damián, (1970), Literatura oral del ladino entre los sefardíes des Oriente a través del romancero, Valencia : Artes Gráficas Soler.

ARMISTEAD, Samuel G., \& SILVERMAN, Joseph H., (1981), Judeo-Spanish Ballads from New York Collected by Mair José Benardete, Berkeley : University of California Press.

GONZALO MAESO, David \& PASCUAL RECUERO, Pascual, (1964), Me'am Lo'ez - El gran comentario bíblico sefardí, Tome preliminaire -Prolegomènes, Madrid : Gredos.

- (1969), M e'am Lo'ez - El gran comentario bíblico sefardí, Tome I, $1^{\circ}$ partie (Gn I-XXV, 18) (sic), Madrid : Gredos.

- (1970), M e’am Lo'ez - El gran comentario bíblico sefardí, Tome I, $2^{\circ}$ partie (Gn I-XXV, $16-\mathrm{L}, 26)$, Madrid : Gredos. 
HEMSI, Alberto, (1995), Cancionero sefardí, E. Seroussi éd., Jérusalem : The Jewish Music Research Centre - The hebrew University.

KOEN-SARANO, Matilda, (1991), Djoha ke dize?, Jérusalem : Kana.

PAPO, Yehudah Eliezer, (1870), Pele Yo'ets, Tome 1, Vienne : Jacob Schlossberg. [Judéoespagnol en caractères Rashi].

PASCUAL RECUERO, Pascual, (1979), Antología de cuentos sefardies, Biblioteca nueva Sefarad, Vol. VI, Barcelone : Ameller.

ROMERO Elena, (1992), La creación literaria en lengua sefardí, Madrid : MAPFRE.

- (2003) Seis coplas sefardies de castiguerio de Hayim Yom-Tob Magula, Madrid : CSIC.

SEPHIHA Haïm Vidal (1992), Du miel au fiel-Contes judéo-espagnols, Paris : Bibliophane.

\section{Corpus espagnol}

ALVAREZ, Guzmán, (1970), Los Proverbios morales de Sem Tob, Salamanca : Biblioteca Anaya.

BIZZARRI Hugo, (2000), Diccionario paremiológico e ideológico de la Edad Media Castilla Siglo XIII, Buenos Aires : Secrit.

Bocados de Oro, cf. CROMBACH, Mechtild.

BONSENYOR, Jafuda, (1990) Libro de palabras y dichos de sabios y filósofos, Introduction et traduction de José Ramón Magdalena Nom De Deu, Biblioteca nueva sefarad, vol. XV, Barcelona: Riopedras.

CORREAS, Gonzalo, (1967), Vocabulario de refranes y frases proverbiales (1627), Louis Combet éd., Institut d'Etudes Ibériques et Ibéro-américaines, Bordeaux.

- (2000) Vocabulario de refranes y frases proverbiales (1627), éd. de L. Combet révisée par R. Jammes et M. Mir-Andreu, Nueva Biblioteca de Erudición y Crítica, Madrid : Castalia.

CROMBACH, Mechtild, (1971), Bocados de Oro, Bonn: Romanischen Seminar des Universitat.

El Libro de los Cien Capítulos, (1960), A. Rey éd., Bloomington : Indiana University Press.

ESPINOSA, Francisco de, (1968), Refranero (1527-1547), E. O'Kane éd., Anejos del Boletín de la Real Academia Española, XVIII, Madrid : Aguirre.

GALMES DE FUENTES, Alvaro, (1991), Dichos de los siete sabios de Grecia- Sentencias morales en verso, Madrid : Gredos. 
GENOT-BISMUTH, Jacqueline-Lise, (2001), Moïse le Séfarade Alias Pierre d'Alphonse La Discipline de Clergie - Disciplina Clericalis, Paris / St Pétersbourg: Ed. de Paris/ Evropeiski Dom.

GONZALEZ-PALENCIA Angel, (éd.), (1948), Disciplina Clericalis - Edición y traducción del texto latino, Madrid : CSIC.

HARO, Marta, (1992), «Dichos e castigos de profetas e filosofos que toda verdad fablaron » in Atalaya, 3, pp. 100 à 138.

KNUST Hermann, (1878) Flores de filosofía, Bibliófilo español, 17.

- (1879) Libro de los buenos proverbios que dixieron los phi(losophos) in Mittheilungen aus dem Eskurial, Bibliothek des litterarischen Verein, 141, Tubingen.

LOPEZ DE MENDOZA, Iñigo, Marqués de Santillana, (1995), Refranes que dizen las viejas tras el fuego, H. O. Bizzarri éd., Kassel : Reichenberger.

LOPEZ GRIGERA, Luisa, (1978) «Un nuevo códice de los Proverbios morales de Sem Tob » in Boletín de la Real Academia española, 56, pp. 221 à 281.

MARTINEZ KLEISER, Luis (1953) Refranero general ideológico español, Madrid : Real Academia Española.

MOREL-FATIO, A. (1878), «El Libro de exenplos por A. B. C. de Climente Sánchez, Archidiacre de Valderas », in Romania, $7^{\circ}$ année, Paris : F. Vieweg, pp. 481 à 526.

NAVARRO SANTIN, Francisco, (1904), «Colección de refranes del siglo XV » in Revista de Archivos, Bibliotecas y Museos, X, pp. 434 à 446.

O'KANE, Eleanor S., (1959), Refranes y frases proverbiales españolas de la Edad Media, Anejos del Boletín de la Real Academia Española (II), Madrid.

PAZ Y MELIA Antonio, éd., (1892) Castigos y doctrina que un sabio dava a sus hijos (S. $X V)$, Bibliófilo español (17), Madrid.

RIUS SERRA J. (1926) : «Refranes del siglo XIV - Romancea Proverbiorum » in Revista de Filología Española, Tome XIII, Madrid, pp. 364 à 372.

Romancea Proverbiorum, cf. COMBET, Louis (1971) et RIUS SERRA, J.

SBARBI, José María, (1980), El Refranero general español - Parte recopilado y parte compuesto, Tome VII, Madrid : A. Gomez Fuentenebro.

STURM H., 1971, The «Libro de los buenos proverbios» - A Critical Edition, The University Press of Kentucky. 


\section{Littérature médiévale judéo-espagnole}

ARRAGEL, Rabi Mosé de Guadalajara, « Noticia de la Biblia puesta en romance 1422 1423 », in Homenaje a Menendez y Pelayo, Madrid, 1899, II, 72.

FONT ESTELLA, Jordi, (1995) «Las sátiras misóginas de Maimón Gallipapa : El voto de la viuda y Querellas de una mujer » in Miscelánea de estudios árabes y hebraicos, sección hebreo, Vol. 44, Univ. de Granada, pp. 149 à 170.

ITZHAKI Masha \& GAREL Michel, (1993) Jardin d'Eden, Jardins d'Espagne, Paris : Seuil/Bibliothèque Nationale.

SAENZ- BADILLOS, Angel \& TARAGONA - BORRAS, Judit (1988), Diccionario de autores judios (Sefarad. Siglos X-XV), Cordoue : El Almendro. 


\section{Parémiologie}

BAŞGÖZ, Ilhan, (1990), «Proverbs about Proverbs or Folk Definitions of Proverb » in Proverbium, 7, pp. 7 à 18.

BELTRAN Rafael, (1997), «Lectura y adaptación de las glosas del Marqués de Santillana a sus Proverbios en la Suma de virtuoso deseo », in Proceedings of the eighth colloquium, A. M. Besresford \& A. Deyermond éd., Papers of the Medieval Hispanic Research Seminar 5, Londres : Dept. of Hispanic Studies, Queen Mary and Westfield College, pp. 49-60.

BIZZARRI Hugo Oscar (1995c) cf. LOPEZ DE MENDOZA.

BIZZARRI, Hugo,(1993) « la palabra y el silencio en la literatura sapiencial de la edad media castellana » in Incipit, 13, pp. 21-49.

- (1992) « Textos sentenciosos de influjo bíblico (ms. BN Madrid 6608)», in Atalaya

- Textes brefs inédits espagnols du Moyen Age, n 3, pp. 93 à 100.

- (1995a) «Oralidad y escritura en el refranero medieval » in Proverbium, $\mathrm{n}^{\circ} 12, \mathrm{pp}$. 27 à 66.

- (1995b) «Deslindes histórico-literarios en torno a Flores de filosofía y Libro de los cien capítulos » in Incipit, 15, pp. 45 à 53.

- (2004) El refranero castellano en la Edad Media, Madrid : Laberinto.

BLECUA, Alberto, (1979), «La littérature apophtegmatique en Espagne » in L'Humanisme dans les Lettres Espagnoles, Etudes réunies et présentées par A. Redondo, Paris : Vrin, pp. 119 à 132.

CANTERA ORTIZ de URBINA, Jesús (2002a) «Aportación cultural del refranero sefardí » in Judaísmo Hispano - Estudios en memoria de José Luis Lacave Riaño, E. Romero éd., Madrid : CSIC, vol. I, pp. 341 à 351.

- (2002b) «La parémiologie judéo-espagnole» in Proverbes et énoncés sentencieux, M. Quitout éd., Paris : L’Harmattan, pp.17 à 32 .

CARRION, Manuel, (1979) «A propósito del elogio al libro de Don Sem Tob de Carrion », in Revista de Archivos, Bibliotecas y Museos, 82, pp. 449 à 460.

CHEVALIER, Maxime, (1975), Cuentecillos tradicionales en la España del Siglo de oro, Madrid : Gredos.

- (1979), «Proverbes, contes folkloriques et historiettes traditionnelles dans les œuvres des humanistes espagnols parémiologues» in L'Humanisme dans les Lettres Espagnoles, Etudes réunies et présentées par A. Redondo, Paris : Vrin, pp. 105 à 118.

COMBET Louis, (1971), Recherches sur le Refranero castillan, Paris : Les Belles Lettres.

CONCA, Maria et GUIA, Josep, (1996) Els primers reculls de proverbis catalans, Barcelona : Publicacions de l'abadia de Montserrat.

DEVOTO, Daniel, (1972) Introducción al estudio de Don Juan Manuel, y en particular de El Conde Lucanor - Una bibliografía, Paris : Ediciones Hispano-Americanas. 
DEYERMOND, Allan D. (1971), A Literary History of Spain - The Middle Ages, Londres : Ernest Benn.

DIAZ-MAS, Paloma, (1993), «Un género casi perdido de la poesía castellana medieval : La clerecía rabínica » in Separata del Boletín de la Real Academia Española, Tomo LXXIII, Cuaderno CLIX, pp.329 à 346.

FRENK ALATORRE, Margit, (1961) «Refranes cantados y cantares proverbializados » in Nueva Revista de Filología Hispánica, tome XV, pp. 155 à 168.

- (1971) «Una fuente poética de Gonzalo Correas » in Nueva Revista de Filología Hispánica, tome XX, pp 90 à 95.

GARCIA GOMEZ, Emilio, (1977) «Una prueba que el refranero árabe fue incorporado en traducción al refranero español », in Al-Andalus, XLII, pp. 375 à 390.

GELLA ITURRIAGA, José, (1978) «Los proverbios del caballero Cifar » in Homenaje a Julio Caro Baroja, Madrid : Centro de Investicaciones Sociológicas, pp. 449 à 464.

GOLDBERG, Harriet, (1993) «The judeo-Spanish Proverb and its Narrative Context" in Publications of the Modern Language Association of America, $\mathrm{n}^{\circ} 108$ (1), pp. 106 à 120.

GONZALEZ LlUBERA, Ignacio, (ed.), (1947), Santob de Carrion - Proverbios morales, Cambridge : Cambridge University Press.

GRANJA (de la), Fernando, (1972), «Del perro de Olías y otros perros » in Al-Andalus, ${ }^{\circ}$ 37, pp. 463-482.

- (1976) «Llenar el ojo » in Al-Andalus, $n^{\circ}$ 41, fasc 2, pp. 445-459.

HABOUCHA, Reginetta, (1992) Types and Motifs of the Judeo-Spanish Folktales, New York \& Londres : Garland.

HASAN-ROKEM, Galit, (1982) Proverbs in Israeli Folk Narratives a Structural Semantic Analysis, Helsinki : Suomalainen Tiedeakatemia

JIGA, Caïus Tiberius, (1976) «Exemples de la typologie sémantique des proverbes dans les langues romanes » in Actes du XIII ${ }^{\circ}$ Congrès international de linguistique et philologie romanes, publiés par M. Boudreault et F. Möhren éd., Québec : Les Presses de l'Université Laval, Vol. 1, pp. 1199-1209.

KANTOR, Sofia, (1992) «Parallelism in the Proverbios morales of Santob de Carrion » in Circa 1492. Proceedings of the Jerusalem Colloquium: Litterae Judaeorum in Terra Hispanica, I. Benabu éd. Isaac, Jérusalem : Hebrew University \& Misgav Yerushalayim, pp 109-133.

LACARRA, Maria Jesús (ed.), (1996) Estudios sobre Pedro Alfonso de Huesca, Huesca : Instituto de Estudios Altoaragoneses.

LE ROUX DE LINCY, M. (1859), Le livre des proverbes français, précédé de Recherches historiques sur les proverbes français dans la littérature du Moyen Age et de la Renaissance, Paris: Adolphe Delahays. 
LEVY Isaac Jack, \& ZUMWALT, Rosemary, (1990) «A Conversation in Proverbs : JudeoSpanish Refranes in Context » in Proverbium, 7, pp. 117 à 132.

- \& ZUMWALT, Rosemary, (1994) «Aun de lo suzio profita il mundo (even from the dirty the world profits) : A Hidden Side of Sephardic Proverbs » in Proverbium, 11, pp. 143 à 158.

MOSCONA Isac, (1973) «On some Problems Connected with the Repetition of Words in the Sayings of the Balkan Jews » in Annual, vol. VIII, pp. 95 à 129.

- (1975) «Proverbs and Sayings of Spanish Origin in the Folklore of the Balkan Jews » in Annual, Vol X, Sofia, pp. 165 à 194.

- (1980) «On the Richness of Sefaradic Folklore. On sefaradic Paremiology » in

Annual, vol. XV, pp. 73 à 102.

- (1981) P $P^{e}$ nine Sefarad, Tel Aviv : Mose Giora Elimelekh.

Neue Romania - Judenspanisch VII, (2003), n 28, Berlin.

OHAYON-BENITHA Pénina, (1991), Contribution à la parémiologie judéo-espagnole: l'Exemple marocain, Aix-en-Provence : Publications de l'Université de Provence.

O'KANE Eleanor, (1970) "What's in a name?" in Proverbium - Archer Taylor octogenario in Honorem I VIII MCMLXX, n 15, pp. 92 à 100.

RATZHABI, Yehudah (1988) «Les sources arabes du Mivhar ha-p ninim » in Sinä̈, ${ }^{\circ}{ }^{\circ} 102$, Jérusalem, pp. 97 à 160. (en hébreu).

ROMERO Elena, (2003) Seis coplas sefardíes de castiguerio de Hayim Yom-Tob Magula, Madrid : CSIC.

ROSENTHAL, Franz (1960-1961), «Al-Mubashshir ibn Fâtik - Prolegomena to an abortive edition » in Oriens, n ${ }^{\circ} 13-14$, pp. 131 à 158.

RUBIERA MATA, Maria-Jesús, (1992), Literatura Hispanoárabe, Madrid : MAPFRE.

RÜGER, Hans Peter, (1984), «Rabbinische Sprichwörter in Spanischem und Judenspanischem Gewande » in Judaica, Vol. 40, n 2, Zürich, pp. 109 à 124.

SADAN, Joseph, (1986), «A Adāb - règles de conduite et $\bar{a} d \bar{a} b$ - dictons, maximes, dans quelques ouvrages inédits d'Al-Ta āâlib̄̄ » in Revue des Etudes Islamiques - Mélanges offerts au Professeur Dominique Sourdel, vol. LIV, pp.285 à 302.

SEPHIHA Haïm Vidal, (1984) «La société judéo-espagnole à travers ses proverbes ou dismoi tes proverbes, je te dirai qui tu es » in Richesse du Proverbe, vol. 2, Actes du colloque de parémiologie (Lille 6-8 mars 1981), F. Suard et C. Buridant éd., Université de Lille, pp. 199 à 209.

$37-47$.

(1990) «L’Humour des Proverbes judéo-espagnols » in Humoresques, n¹, pp. 
SEVILLA MUŇOZ, Julia (2002) « La parémiologie (comparée) espagnole à l'aube du XXI siècle » in Proverbes et énoncés sentencieux, M. Quitout (éd.) Paris : l'Harmattan.

SCHULZE-BUSACKER Elisabeth, Proverbes et expressions proverbiales dans la littérature narrative du Moyen-Age français - Recueil et analyse Paris : Champion, 1985.

TAYLOR, Archer, The proverb and an Index to the Proverb, 1962

TAYLOR Barry, (1985) «Old Spanish Wisdom Texts: Some Relationships» in La Corónica, 14, 1985, pp. 71 à 85.

- (1992) «Wisdom Forms in the Disciplina Clericalis of Petrus Alfonsi », in Circa 1492- Proceedings of the Jerusalem Colloquium : Litterae Judaeorum in Terra Hispanica, I. Benabu éd., Jérusalem : The Hebrew University / Misgav Yerushalayim, pp. 175 à 188.

- (1996) «La sabiduría de Pedro Alfonso : La Disciplina Clericalis », in Lacarra María-Jesús (cf supra), pp. $291-308$

THOMPSON, John Mark, (1974), The Form and Function of Proverbs in Ancient Israel, La Haye - Paris : Mouton.

TOLAN John, (1993) Petrus Alphonsi ans his Medieval Readers, Gainesville : University Press of Florida.

TUBACH, Frédéric C., (1969) Index Exemplorum : a Handbook of Medieval Religious Tales, Helsinki : Suomalainen tiedeakatemia.

VAROL (BORNES), Marie-Christine, (1996) «Djohá juif, dans les proverbes et les contes judéo-espagnols » in L'Humour en Orient, REvue du Monde Musulman et de la Méditerranée (REMMM), n 77 - 78, I. Fenoglio et F. Georgeon éd., Paris : Edisud, pp. 60 à 74.

- (1999) «Raíces medievales de los proverbios judeo-españoles» in Actes des Journées judéo-espagnoles de Londres, A. Benaïm ed., Londres : Queen Mary and Westfield college, pp. 203 à 218.

- (2000) «Le passage d'une langue à l'autre des expressions imagées du judéoespagnol de Turquie: Los mijores de mozós, ahuera las karas, la novya de las syete fustanelas, et autres énigmes » in Cahiers du Prohemio, $\mathrm{n}^{\circ} 3$, A. Iglesias Ovejero éd., Orléans : Presses universitaires, pp. $409-424$.

- (2001) «Les Judéo-Espagnols de Turquie et le discours indirect» in Hommage à Marthe Westphal, R. Caplán, M. L. Copete \& I. Reck éds., Nancy : Institut d'espagnol et de Portugais, pp. 151- 179.

VERNET Juan, (1985) Ce que la Culture doit aux Arabes d'Espagne, Paris : Sindbad (2 edition de la traduction française de La cultura hispanoárabe en Oriente y Occidente, 1978) .

WACHTEL, Nathan (2001), La Foi du souvenir-Labyrinthes marranes, Paris : Seuil.

WALSH, John K. (1976), «Versiones peninsulares del Kitāb ādāb al-falāsifa de Hunayn Ibn Ishāq - Hacia una reconstrucción del Libro de los buenos proverbios» in Al-Andalus, tome 41 , fasc. 2 , pp. 355 à 384. 
WAGNER, Max Leopold, (1990) Sondersprachen der Romania III \& IV - Judenspanish I \& II, réed. par H. Kröll de l'ensemble de ses travaux, ( 2 vol.), Stuttgart : Franz Steiner

WEBSTER Sheila K., (1986) «Arabic Proverbs and Related Forms » in Proverbium, $\mathrm{n}^{\circ} 3$, pp. 179 à 194.

ZEMKE, John, (1997a) «Shem Tov de Carrion's Proverbios morales: A sermon addressed, in Romance Philology, Vol. LI, 2, University of California Press, pp. 194 à 210.

- (1997b) Critical Approaches to the Proverbios morales of Shem Tov de Carrión : An annotated Bibliography, Newark : Juan de la Cuesta.

\section{ANNEXES}

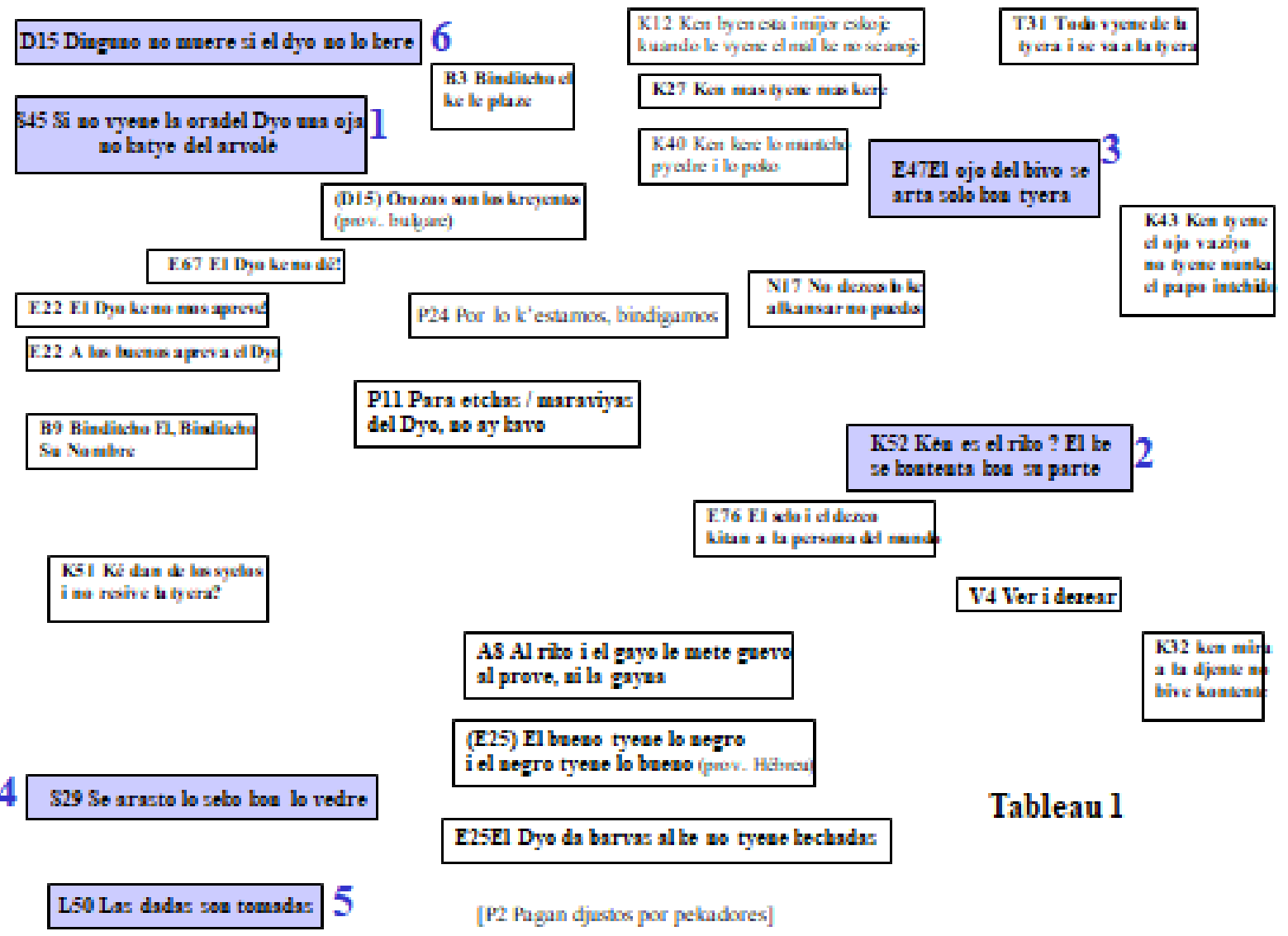

\section{Tableau 1}




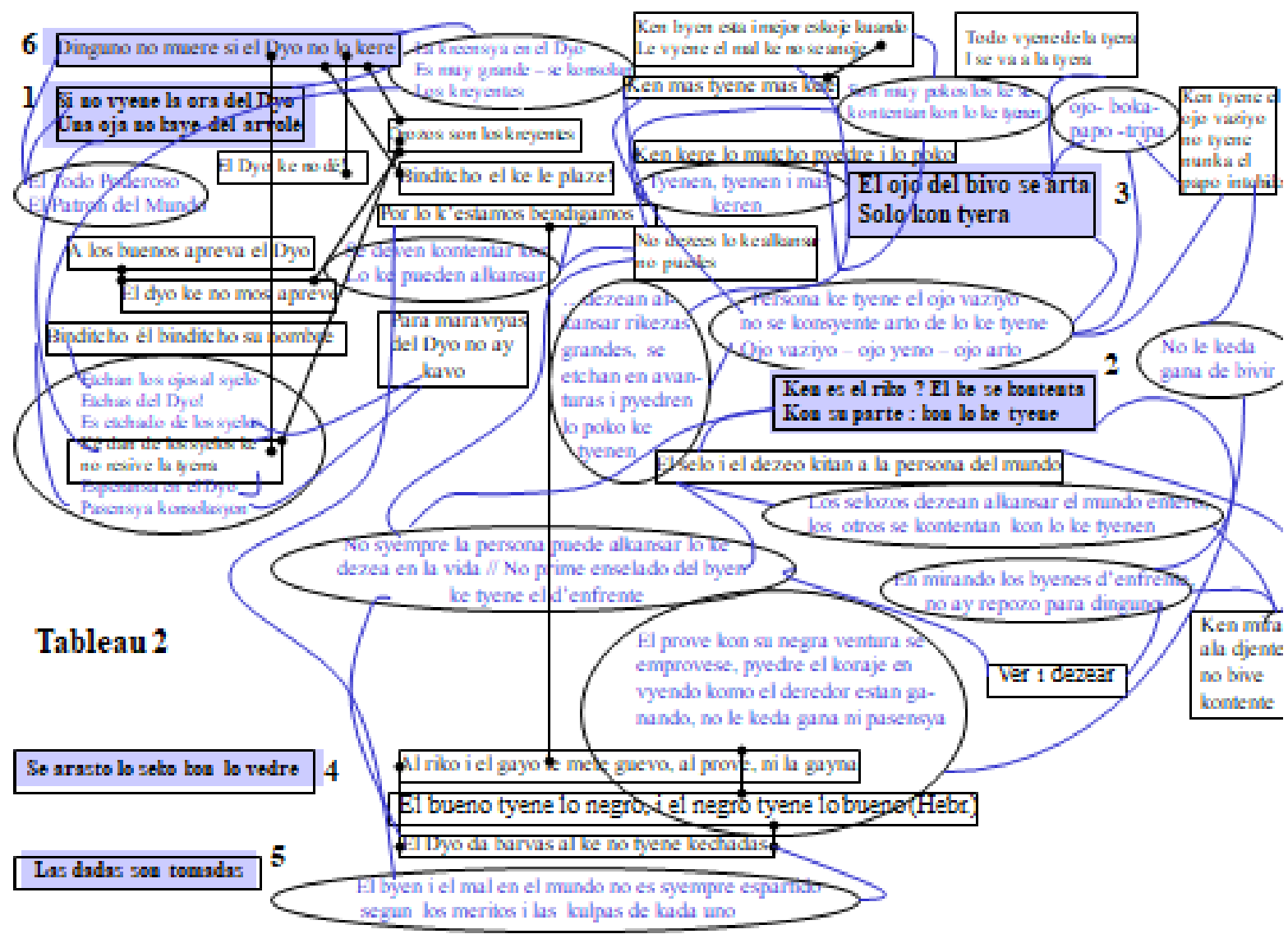

[Pagan djestor por peladores]

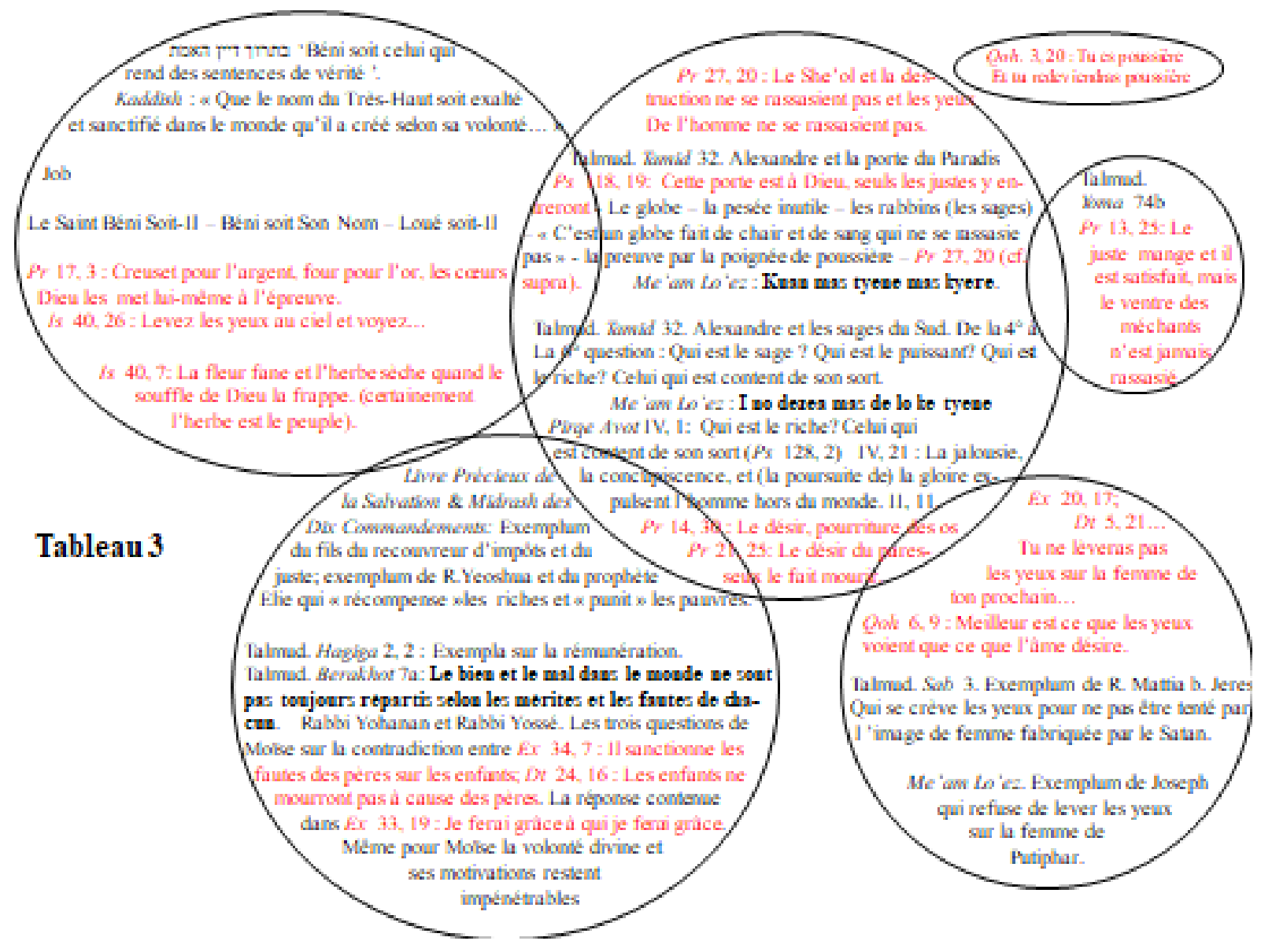


I no saves que las condas de la mar no se mecen sino con I 'aire que manda Allah sobre ellar [...] lo loc ramos de loc arbeles no se mecen sies con l'sire este y las yembs no hacen vingen coca sieo por el mandsmiento del Criador

Entrevue avec Dindimus

Ngradece a Allah bo que ie da Tetre d'A lexandre i A ristote sur les

Nestros padres agradecian bo ke ks ea dady

Nos combentamos bon bo be ienemos de esito
Y to toramiento sera a aquesta tierr

y no podras por menos de tornar a aque-

[palmo de tierra]

La source du Paradis

Aci como no se farta squesta piedra

(...) sai wuca to fartara to ojo do

vingers cocs... Y Iless ta ojo merveilles de 1 'Tude

I iodo bo ke has ganado i has encenomado (...) i has a llegado, bo dexaras

Alexandre et les Gymmosophistes

Basiete con bo que vives dia empoés de dia; y te hartara bo poco si ie conformas con ello

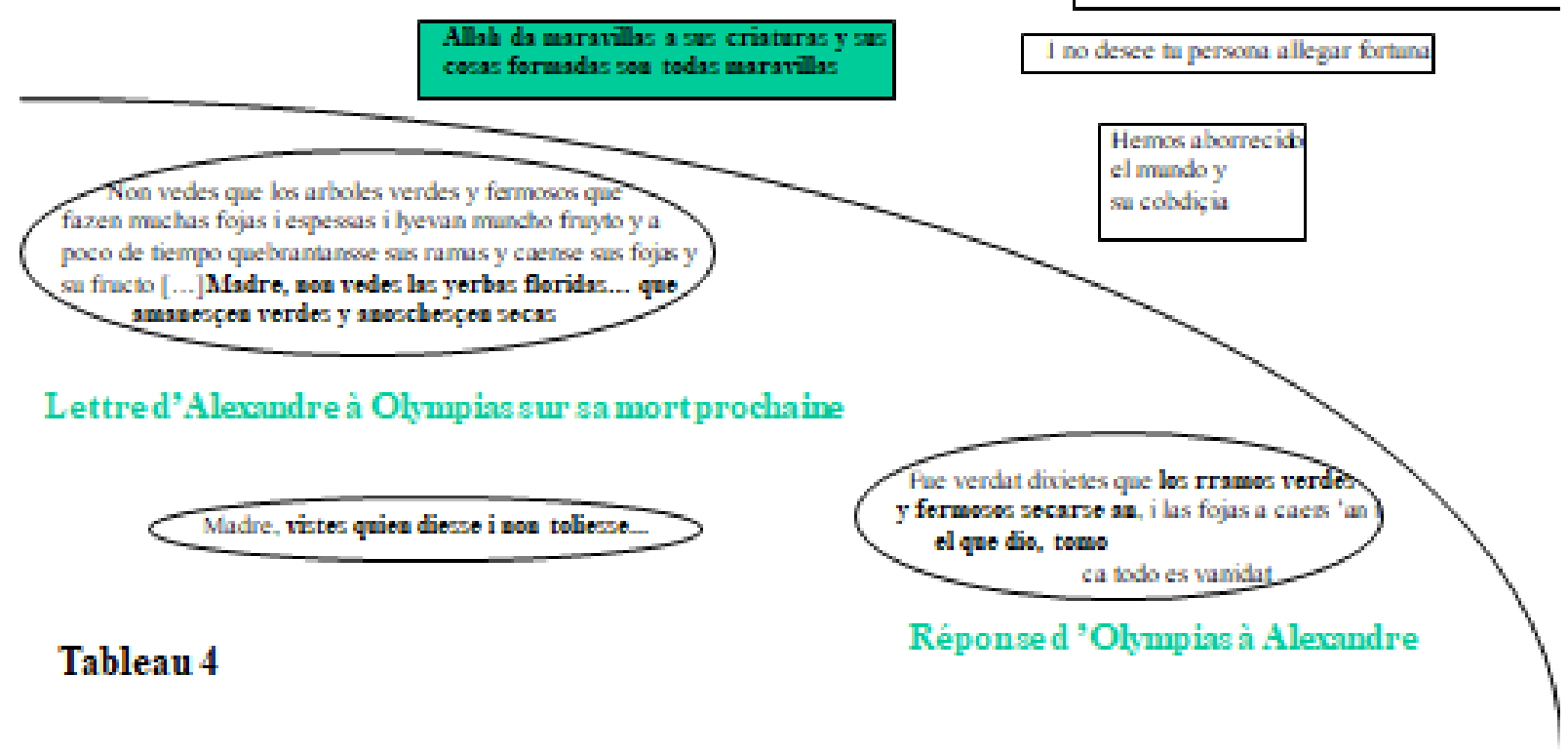

